\title{
A Revised Assessment of Calibration Uncertainties for Capsule Type Standard Platinum and Rhodium-Iron Resistance Thermometers
}

\author{
W. L. Tew \\ G. F. Strouse \\ C. W. Meyer
}

U.S. DEPARTMENT OF COMMERCE
Technology Administration
Process Measurements Division
National Institute of Standards
and Technology
Gaithersburg, MD 20899-0001

$Q C$ 100 .056 N0.6138 1998 



\title{
A Revised Assessment of Calibration Uncertainties for Capsule Type Standard Platinum and Rhodium-Iron Resistance Thermometers
}

\author{
W. L. Tew \\ G. F. Strouse \\ C. W. Meyer
}

U.S. DEPARTMENT OF COMMERCE Technology Administration Process Measurements Division National Institute of Standards and Technology

Gaithersburg, MD 20899-0001

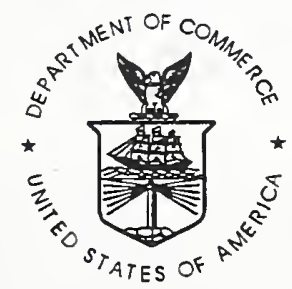

U.S. DEPARTMENT OF COMMERCE William M. Daley, Secretary

TECHNOLOGY ADMINISTRATION Gary R. Bachula, Acting Under Secretary for Technology

NATIONAL INSTITUTE OF STANDARDS

Raymond G. Kammer, Director 



\title{
A Revised Assessment of Calibration Uncertainties for Capsule Type Standard Platinum and Rhodium-Iron Resistance Thermometers
}

\author{
W. L. Tew, G.F. Strouse and C. W. Meyer \\ National Institute of Standards and Technology \\ Process Measurements Division \\ Gaithersburg, MD 20899
}

\section{Introduction}

The International Temperature Scale of 1990 (ITS-90) |1] came into effect on 1 January 1990, superseding the International Practical Temperature Scale of 1968. Amended Edition of 1975 [2] and the 1976 Provisional $0.5 \mathrm{~K}$ to $30 \mathrm{~K}$ Temperature Scale [3]. The ITS-90 extends upward from $0.65 \mathrm{~K}$, and is defined by equilibrium states of pure substances (defining thermometric fixed points), interpolating devices, and equations of state that relate the measured property to $T_{90}$.

The National Institute of Standards and Technology (NIST) provides calibration services for resistance thermometers over all the ITS-90 temperature subranges [4]. There are eleven subranges defined for standard platinum resistance thermometers (SPRTs), covering temperatures between $13.8033 \mathrm{~K}$ and $1234.93 \mathrm{~K}$. For SPRTs of the capsule type, only the seven lowest subranges of these eleven are normally used for calibration (see Table 1). In addition, the ITS-90 defines a set of vapor pressure (VP) relationships for ${ }^{3} \mathrm{He}$ and ${ }^{4} \mathrm{He}$ in the range between $0.65 \mathrm{~K}$ and $5.0 \mathrm{~K}$ and a set of ${ }^{3} \mathrm{He}$ and ${ }^{4} \mathrm{He}$ gas pressure relationships between $3.0 \mathrm{~K}$ and $24.5561 \mathrm{~K}$ using an interpolating constant volume gas thermometer (ICVGT). Calibrations on the ITS-90 are provided for these two lowest ranges, but these can be accomplished only by transferring the scale to capsule type rhodium-iron resistance thermometers (RIRTs) [5].

In January of 1994 an internal report, NISTIR 5319 [6], was issued which described the new NIST method of expressing measurement uncertainty according to the guidelines adopted by NIST [7] and the International Organization for Standardization (ISO) [8]. This report described the calibration uncertainties for all types of SPRTs and RIRTs, based on the calibration methods used and realizations performed at that time. Since then, a number of significant advances in ITS-90 realizations and calibration techniques below $83.8 \mathrm{~K}$ have taken place at NIST. Perhaps the most significant of these advances is that, starting with calibration reports issued in October 1996 or later, an "as defined" ITS-90 is being disseminated below $83.8 \mathrm{~K}[9 \mid$. This replaces the "wire scale" version of the scale. ITS-90(W), which had been disseminated from January 1990 to September 1996 [10] for the range $0.65 \mathrm{~K}$ to $83.8058 \mathrm{~K}$.

These advances have necessitated a revision of the earlier assessment of uncertainties as given in NISTIR 5319. This revision applies only to capsule SPRTs that are suitable as defining interpolating devices of ITS- 90 and to capsule RIRTs that are suitable as high-quality scale-transfer devices, and only to those when used below $83.8 \mathrm{~K}$. All other types of SPRTs described in NISTIR 5319 are unaffected by this revision since all of those are calibrated exclusively above $83.8 \mathrm{~K}$. 


\section{Calibration Procedures}

At NIST, calibrations of all types of SPRTs for subranges above $83.8 \mathrm{~K}$ are accomplished via direct measurement at thermometric fixed points of the ITS-90. Calibrations of SPRTs below $83.8 \mathrm{~K}$ are accomplished via direct comparison with designated reference SPRTs and are limited to capsule type SPRTs. These reference SPRTs are calibrated at the ITS-90 fixed points given in the first range of Table I ( $13.8033 \mathrm{~K}$ to $0.01^{\circ} \mathrm{C}$ ). Likewise, calibrations of capsule RIRTs below $83.8 \mathrm{~K}$ are performed via direct comparison with combinations of designated reference SPRTs and reference RIRTs.

Table I. Subranges of the ITS-90 which are appropriate for the calibration of capsule SPRTs.

Temperature Subrange

\begin{tabular}{|c|c|c|c|}
\hline $13.8033 \mathrm{~K}$ & to & $0.01{ }^{\circ} \mathrm{C}$ & $\begin{array}{l}\mathrm{e}-\mathrm{H}_{2}(\mathrm{TP}), \mathrm{e}-\mathrm{H}_{2}(\mathrm{VP} \text { at } 17 \mathrm{~K}), \mathrm{e}-\mathrm{H}_{2}(\mathrm{VP} \text { at } 20.3 \mathrm{~K}), \\
\mathrm{Ne}(\mathrm{TP}), \mathrm{O}_{2}(\mathrm{TP}), \mathrm{Ar}(\mathrm{TP}), \mathrm{Hg}(\mathrm{TP}), \mathrm{H}_{2} \mathrm{O}(\mathrm{TP})\end{array}$ \\
\hline $24.5561 \mathrm{~K}$ & to & $0.01{ }^{\circ} \mathrm{C}$ & $\begin{array}{l}\text { e- } \mathrm{H}_{2}(\mathrm{TP}), \mathrm{Ne}(\mathrm{TP}), \mathrm{O}_{2}(\mathrm{TP}), \quad \mathrm{Ar}(\mathrm{TP}) \\
\mathrm{Hg}(\mathrm{TP}), \mathrm{H}_{2} \mathrm{O}(\mathrm{TP})\end{array}$ \\
\hline $54.3584 \mathrm{~K}$ & to & $0.01^{\circ} \mathrm{C}$ & $\mathrm{O}_{2}(\mathrm{TP}), \operatorname{Ar}(\mathrm{TP}), \mathrm{Hg}(\mathrm{TP}), \mathrm{H}_{2} \mathrm{O}(\mathrm{TP})$ \\
\hline $83.8058 \mathrm{~K}$ & to & $0.01{ }^{\circ} \mathrm{C}$ & $\mathrm{Ar}(\mathrm{TP}), \mathrm{Hg}(\mathrm{TP}), \mathrm{H}_{2} \mathrm{O}(\mathrm{TP})$ \\
\hline $34.3156 \mathrm{~K}$ & to & $29.7646^{\circ} \mathrm{C}$ & $\mathrm{Hg}(\mathrm{TP}), \mathrm{H}_{2} \mathrm{O}(\mathrm{TP}), \mathrm{Ga}(\mathrm{MP})$ \\
\hline $0{ }^{\circ} \mathrm{C}$ & to & $29.7646^{\circ} \mathrm{C}$ & $\mathrm{Ga}(\mathrm{MP}), \mathrm{H}_{2} \mathrm{O}(\mathrm{TP})$ \\
\hline $0^{\circ} \mathrm{C}$ & to & $156.5985^{\circ} \mathrm{C}$ & In (FP), $\mathrm{H}_{2} \mathrm{O}(\mathrm{TP})$ \\
\hline $0^{\circ} \mathrm{C}$ & to & $231.928^{\circ} \mathrm{C} \dagger$ & $\mathrm{Sn}(\mathrm{FP}), \mathrm{In}(\mathrm{FP}), \mathrm{H}_{2} \mathrm{O}(\mathrm{TP})$ \\
\hline
\end{tabular}

$\uparrow$ This temperature may be too high for some capsule SPRTs, and calibration over this sub-range is generally not recommended.

$\mathrm{VP}=$ Vapor Pressure, $\mathrm{TP}=$ Triple Point, $\mathrm{FP}=$ Freezing Point and $\mathrm{MP}=$ Melting Point

2.1 SPRTs. Calibration data for SPRTs consist of resistance ratios, $W\left(T_{90}\right) \equiv R\left(T_{90}\right) / R(273.16 \mathrm{~K})$, which are derived from measurements of the SPRT resistance $R\left(T_{90}\right)$ at the appropriate fixed point temperatures together with the resistance at the triple point of water $R(273.16 \mathrm{~K})$. These values for $W\left(T_{90}\right)$ are then fitted to the pertinent ITS-90 deviation function [4] for the calibration subrange of interest. Measurements at temperatures between the required defining fixed-point temperatures allow checks on the interpolation at these redundant points.

2.2 RIRTs. Calibration data for RIRTs consist of resistance values, $R\left(T_{90}\right)$, taken at a sufficient number of calibration points to construct an accurate interpolation function for the range of interest. Since the RIRT is not a defining instrument of the ITS-90, there is no officially adopted reference 
function for interpolation. A simple polynomial expansion in $T_{90}$, however, is usually sufficient to represent the ITS-90 in the range $0.65 \mathrm{~K}$ to $24.6651 \mathrm{~K}$ [11]. It is possible to calibrate RIRTs at higher temperatures also and such extended calibrations are offered at NIST for temperatures as high as $83.8058 \mathrm{~K}$.

For RIRTs of the nominal composition (atomic fraction: $0.5 \% \mathrm{Fe}, 99.5 \% \mathrm{Rh}$ ), the $R\left(T_{90}\right)$ curve has an inflection point close to $27 \mathrm{~K}$. This is usually an optimal break point for curve fitting. A typical set of calibration points is shown in Table II for a standard range calibration $(0.65 \mathrm{~K}$ to $27.1 \mathrm{~K})$ and for an extended range calibration $(0.65 \mathrm{~K}$ to $83.8058 \mathrm{~K})$. These points are of a sufficient number and spacing for fitting a polynomial function to the data. If an additional breakpoint is made at $5.1 \mathrm{~K}$, then two separate seventh order polynomials will usually yield interpolation inaccuracies of no greater than $0.1 \mathrm{mK}$ below $5 \mathrm{~K}$ and $0.2 \mathrm{mK}$ between $5 \mathrm{~K}$ and $27 \mathrm{~K}$. A sixth order polynomial for the extended range of $26 \mathrm{~K}$ to $83.8 \mathrm{~K}$ will usually yield interpolation inaccuracies no greater than $0.1 \mathrm{~K}$. These calibration points include the secondary reference point (undefined by ITS-90) temperatures for four of the superconducting transition points of NIST Standard Reference Material 767 [12], as well as the six ITS-90 defining fixed points that lie in the range below $83.8 \mathrm{~K}$.

Table II. RIRT calibration point (\#) spacing for a 25-point standard range calibration between $0.65 \mathrm{~K}$ and $27.1 \mathrm{~K}$ and for a 37-point extended range calibration between $0.65 \mathrm{~K}$ and $83.8 \mathrm{~K}$.

\begin{tabular}{|c|c|c|c|c|c|c|c|}
\hline \hline$\#$ & $T / \mathbf{K}$ & $\#$ & $T / \mathbf{K}$ & $\#$ & $T / \mathbf{K}$ & $\#$ & $T / \mathbf{K}$ \\
\hline 1 & 0.65 & 2 & 0.85 & 3 & 1.181 & 4 & 1.65 \\
\hline 5 & 2.2 & 6 & 2.8 & 7 & 3.4145 & 8 & 4.2 \\
\hline 9 & 5.1 & 10 & 6.1 & 11 & 7.1997 & 12 & 8.6 \\
\hline 13 & 10.2 & 14 & 12.0 & 15 & 13.8033 & 16 & 15.4 \\
\hline 17 & 17.036 & 18 & 18.66 & 19 & 20.271 & 20 & 21.5 \\
\hline 21 & 22.6 & 22 & 23.6 & 23 & 24.5561 & 24 & 26.0 \\
\hline 25 & 27.1 & & & & & & \\
\hline
\end{tabular}

\begin{tabular}{|c|c|c|c|c|c|c|c|}
\hline 24 & 26.0 & 25 & 27.1 & 26 & 31.2 & 27 & 35.3 \\
\hline 28 & 39.4 & 29 & 45.0 & 30 & 50.0 & 31 & 54.3584 \\
\hline 32 & 60.0 & 33 & 65.0 & 34 & 70.0 & 35 & 75.0 \\
\hline 36 & 80.0 & 37 & 83.8058 & & & & \\
\hline
\end{tabular}


2.3 Resistance standards. The individual resistance measurements are actually measurements of resistance ratios, $r_{q j}\left(T_{90}\right) \equiv R_{q}\left(T_{90}\right) / R_{j}$, for a thermometer " $q^{\prime \prime}$ and a standard resistor " $j$ ". These standard resistors generally have a nominal resistance of either $1 \Omega, 10 \Omega$ or $100 \Omega$ and are maintained at a temperature of $25^{\circ} \mathrm{C} \pm 0.01^{\circ} \mathrm{C}$. In addition, the NIST Thermometry Group uses a transportable $25 \Omega$ standard resistor, maintained at $27.7^{\circ} \mathrm{C}$, for the purposes of transferring resistance values between laboratories. All of these resistance standards are of the Wilkins type [13], with ac/dc transfer errors of less than $1 \times 10^{-6}$ of the resistance value. For SPRT calibrations, certain subranges may require only one resistance standard, in which case no special knowledge of the standard is needed for the measurement of $W\left(T_{90}\right)$ and the uncertainty in $R_{j}$ does not contribute to the uncertainty in $W\left(T_{90}\right)$. However, for all SPRT subranges below $83.8 \mathrm{~K}$ this is not the case, as more than one standard is needed when the SPRT resistance decreases by more than two orders of magnitude in going from $83.8 \mathrm{~K}$ to $13.8 \mathrm{~K}$. In this case, a known relationship between the different resistance standards used must be established, and the associated uncertainties in these relationships contribute to the overall calibration uncertainty. In the case of RIRTs, calibrations are reported directly in ohms, and uncertainty in the calibration of the resistance standards always contributes to the uncertainty in the thermometer calibration. When these resistance standards are properly calibrated, however, these uncertainties generally make a minor contribution to the overall uncertainty.

\section{Realization Methods}

Fixed point realizations that are appropriate for capsule SPRT or RIRT calibrations are performed using one of the following three different techniques at NIST. The complexity of the ITS-90 definitions, particularly for those ranges below $24.5561 \mathrm{~K}$, together with the daily demands of providing customer calibrations, has necessitated the separation of these three methods into three separate laboratory facilities within NIST. This separation of laboratories, with three independent measurement systems, especially complicates the evaluation of calibration uncertainties for capsule thermometers, as we will see in section 6.

3.1 Long-stem fixed point cells. For the Ar triple point (TP), $\mathrm{Hg} \mathrm{TP}, \mathrm{H}_{2} \mathrm{O} \mathrm{TP}$, Ga melting point (MP), In freezing point (FP), and Sn FP, special fixed-point cells are used which are designed to be accessed via insertion of either extension probes with capsule SPRTs or long-stem SPRTs [14, 15]. These extension probes contain the capsule SPRT in metal or glass sheaths which are backfilled with He gas. The extension probes of the metal type can accommodate any variety of capsule SPRT and have an outer diameter of $11.1 \mathrm{~mm}$. In this way, the capsule SPRTs may be treated in the same manner as long-stem SPRTs, except that some cells must be constructed with larger re-entrant wells. The extension probes of the glass type are made in various sizes having outer diameters between 7.0 $\mathrm{mm}$ and $7.5 \mathrm{~mm}$. These are specially designed to accommodate certain models of capsule SPRTs

'This notation is chosen to clarify the distinction between a thermometer's resistances and the standard resistor's resistances. The index " $j$ " is primarily intended to distinguish resistance standards of different values (e.g. $1 \Omega, 10 \Omega$, etc.) while the index " $q$ " uniquely identifies each thermometer (such as a serial number) in a batch which may all have similar resistance values at a given temperature . 
with uniform diameters of $5.7 \mathrm{~mm}$ or less. These glass extension probes have the advantage that they may be used in the same fixed point cells that are used for long-stem SPRTs.

For the $\mathrm{Hg} \mathrm{TP}$ and all higher temperature fixed points, these "long-stem" fixed-point cells are the only types available at NIST. Since all of the lower SPRT subranges of the ITS-90 are defined with the water triple point as an upper calibration point, all capsule SPRT calibrations must involve at least some (e.g., Hg TP and $\mathrm{H}_{2} \mathrm{O}$ TP ) fixed-point realizations performed in this manner. At NIST, these long-stem fixed-point cells are used and maintained primarily in the SPRT Calibration Laboratory |16| (LAB A).

3.2 Integrated Realization Block. All of the fixed points of e- $\mathrm{H}_{2}$ (TP), e- $\mathrm{H}_{2}$ (VP at $17 \mathrm{~K}$ ), e- $\mathrm{H}_{2}$ (VP at $20.3 \mathrm{~K}$ ), $\mathrm{Ne}(\mathrm{TP}), \mathrm{O}_{2}$ (TP), and $\mathrm{Ar}$ (TP) may be realized in a single block realization system. At NIST, this system contains a series of six separate fixed point cells and one interpolating constant volume gas thermometer (ICVGT) integrated into a single copper block, with a dedicated cryostat [17], operated and maintained in a separate Low Temperature Realization Laboratory (LAB B). The cells for $\mathrm{Ar}$ and $\mathrm{O}_{2}$ have volumes of $20 \mathrm{~cm}^{3}$ each, while the cells dedicated to $\mathrm{Ne}, \mathrm{e}-\mathrm{H}_{2},{ }^{4} \mathrm{He}$, and ${ }^{3} \mathrm{He}$ have volumes of $3 \mathrm{~cm}^{3}$ each. The block can accommodate two capsule SPRTs and four capsule RIRTs. These thermometer wells are integral with the copper block but not directly in contact with liquid/solid interfaces within the fixed point cells. Pressure measurement capability includes capacitance diaphragm gauges and piston gauges which are integrated into the system. This system has the advantage that an entire sequence of ITS -90 realizations may be performed for any subrange below $83.8 \mathrm{~K}$ on a set of thermometers without having to remove or handle any of the thermometers. Additional details on this realization system and recent realization results are given in references [18] and $[19]$.

3.3 Sealed-Cell Fixed-Point Devices. The fixed points of e- $\mathrm{H}_{2}$ (TP), $\mathrm{Ne}$ (TP), $\mathrm{O}_{2}$ (TP), and $\mathrm{Ar}$ (TP) also may be realized via the sealed-cell method [20]. This method employs permanently sealed samples of material in small, transportable cells that can accommodate up to three capsule thermometers each. At NIST, these fixed point realizations are performed in the Low Temperature Calibration Laboratory (LAB C). This approach has the advantage that the same sample can be maintained, transported, and periodically used for fixed-point realizations over long time periods independent of the cryostat or measurement system used.

\section{Batch Comparisons}

Calibrations of capsule RIRTs and capsule SPRTs below $83.8 \mathrm{~K}$ are performed by comparison in a multiple thermometer batch process. The batch calibration process uses a copper comparison block, in vacuum, that can hold as many as twenty capsule thermometers. At least two of each type (SPRT and RIRT) are designated as reference thermometers. The SPRT reference thermometers are chosen from a set of stable capsule SPRTs maintained at NIST and used in previous fixed-point realizations via some combination of the above three techniques. Similarly, the RIRT reference thermometers are chosen from calibrated sets of RIRTs using previous realization data obtained in LAB B. A single reference resistance thermometer (RRT) from each set is selected to determine the block temperature 
for calibration purposes and the other reference thermometers then serve as check thermometers. These comparison calibrations are performed at NIST within LAB C[21].

During a batch calibration process, the resistance ratio of each of the $n$ uncalibrated batch resistance thermometers, $\mathrm{BRT}_{q}(i)$, where $q=1$ to $n$, are measured at two currents, $i=i_{1}$ and $i=i_{2}$. The values of the measurement currents depend on both temperature and the device resistance (e.g., $25.5 \Omega$ or $100 \Omega$ at $273.16 \mathrm{~K})$. A single measurement of either the RRT or the $\mathrm{BRT}_{q}(i)$ is calculated as an average of 20 or more readings of the ac resistance ratio for that thermometer. A sequence of measurements of the reference thermometer is made in conjunction with measurements of the qth batch thermometer in the following symmetrical order: RRT, $\mathrm{BRT}_{q}\left(i_{1}\right), \mathrm{BRT}_{q}\left(i_{2}\right), \mathrm{BRT}_{q}\left(i_{1}\right), \mathrm{RRT}$. A single temperature is then assigned to the qth thermometer's resistance based on the average ratio value calculated from the two measurements of the RRT.

\section{Measurement Techniques}

\subsection{SPRTs}

The subtleties involved in constructing accurate $W\left(T_{90}\right)$ values in the subranges below $83.8 \mathrm{~K}$, as first mentioned in section 2.3, will now be discussed in detail. The normal practice in operating an ac resistance bridge is to switch to lower resistance standards as the resistance of the test thermometer decreases with decreasing temperature. In this way, the system's measurement resolution, in terms of ratio measurements, remains reasonably constant. Establishing an accurate relationship between all of the resistance standards used in the calibration process then becomes necessary. This may be done in one of two ways: a) "Ratio Chain Method" - ratios may be measured for each of the standards relative to the standard used in the measurement of the triple point of water (usually a $100 \Omega$ standard for capsule SPRTs), or b) "Calibrated Standards Method" - absolute calibrations, in ohms, may be obtained for each of the standards used. There is also an added complication for establishing ratio chains when the measurement systems are separated in two or more laboratories, as they are at NIST.

5.1.1 Ratio Chain Method: A general expression for constructing $W\left(T_{90}\right)$ values can be written as follows,

$$
W_{q}\left(T_{90}\right)=r_{q, m}\left(T_{90}\right) r_{1, q}\left(T_{\text {tpw }}\right) \prod_{j=2}^{m} r_{j, j-1}
$$

where $r_{j, j-1} \equiv R / R_{j-1}$ and where $r_{q, m}\left(T_{90}\right)$ refers to the ratio of resistance $R_{q}\left(T_{90}\right)$ of a thermometer " $q$ " to the resistance $R_{m}$ of a standard resistor " $m$ ". The resistance standards are labeled $j=1$ to $m$, where $m$ depends on the subrange being used and on the number of measurement systems involved in the calibration, as will be seen below. It is important to remember that each $r_{j, m}$ or $r_{q, m}\left(T_{90}\right)$ represents an independent ratio measurement, at least as far as Type A uncertainties are concerned. Also, in 
practice, it is the inverse ratio, $r_{q, 1}\left(T_{\mathrm{tpw}}\right)=1 / r_{\mathrm{l}, q}\left(T_{\mathrm{tpw}}\right)$ that is actually measured $\left(T_{\mathrm{tpw}}=273.16 \mathrm{~K}\right)$, but this detail is irrelevant as far as calculating uncertainties is concerned.

The following examples are constructed for the specific case of a $25.5 \Omega$ (at $T=273.16 \mathrm{~K}$ ) capsule SPRT and ratio measurements made with an ac resistance bridge having a maximum ratio capacity of 1.29. This reflects the present calibration conditions that are customary at NIST. Other arrangements, such as wider range bridges and $100 \Omega$ capsules, would yield different results.

5.1.1.1 Single measurement system: It is standard practice in the use of ac resistance ratio bridges to maintain a set of three standard resistors, under temperature control. Let a single set of $100 \Omega, 10 \Omega$, and $1 \Omega$ standards be labeled by $j=1,2,3$, respectively. For calibration subranges whose low temperature terminations are at temperatures of $83.8 \mathrm{~K}$ or higher, it is customary to use only a single $100 \Omega$ resistance standard for capsule SPRTs, in which case equation 1 reduces to

$$
W_{q}\left(T_{90}\right)=r_{q, 1}\left(T_{90}\right) r_{1, q}\left(T_{\mathrm{tpw}}\right)
$$

For the calibration subranges whose low temperature terminations are at the $\mathrm{O}_{2} \mathrm{TP}$, it is customary to use a $10 \Omega$ resistance standard for all ratio measurements of the SPRT at $83.8 \mathrm{~K}$ and below. In this case, equation 1 then becomes

$$
W_{q}\left(T_{90}\right)=r_{q, 2}\left(T_{90}\right) r_{1, q}\left(T_{\mathrm{tpw}}\right) r_{2,1}
$$

For the calibration subranges whose low temperature terminations are at the Ne TP and the e- $\mathrm{H}_{2} \mathrm{TP}$, it is customary to use a $1 \Omega$ resistance standard for all ratio measurements of the SPRT below roughly $39.4 \mathrm{~K}$ and a $10 \Omega$ resistance standard for all ratio measurements above that point. The temperature of $39.4 \mathrm{~K}$ is approximately midway between the Ne TP and the $\mathrm{O}_{2} \mathrm{TP}$ and it is used as an interpolation check point. A $25.5 \Omega$ (at $\mathrm{H}_{2} \mathrm{O}$ TP) capsule SPRT will have a resistance of approximately $1.02 \Omega$ at $39.4 \mathrm{~K}$, and it is customary to measure this ratio with both $1 \Omega$ and $10 \Omega$ resistance standards to check the consistency of the results as well. Thus, for temperatures of $39.4 \mathrm{~K}$ and below, equation 1 becomes

$$
W_{q}\left(T_{90}\right)=r_{q, 3}\left(T_{90}\right) r_{1, q}\left(T_{\mathrm{tpw}}\right) r_{2,1} r_{3,2}
$$

While it is perhaps simpler to substitute the single ratio measurement of $r_{3,1}$ for the product of $r_{3,2} r_{2,1}$, the loss of resolution in measuring a ratio as small as 0.01 is slightly more severe than combining the uncertainties of two independent ratio measurements of 0.1 each. 
5.1.1.2 Dual measurement systems: There are often limitations on the amount of equipment that can fit into one laboratory. Consequently, this may require separate measurement systems being involved in the calibration of a single SPRT, as is the case with all capsule SPRT calibrations at NIST. In order to maintain the ratio chain, a portable transfer standard must be carried periodically between the two or more laboratories. At NIST, a $25 \Omega$ standard resistor in a transportable, temperature-controlled enclosure serves this purpose. Let the set of resistance standards consisting of a $100 \Omega$ (LAB A), a $25 \Omega$ (portable), a $10 \Omega$ (LAB C), and a $1 \Omega$ (LAB C) resistor, be designated by the indices $j=1,2,3,4$, respectively. The $100 \Omega$ standard is maintained in laboratory A, the $10 \Omega$ and $1 \Omega$ standards are maintained in laboratory $\mathrm{C}$ and the portable transfer standard links the two labs together. For the case of the calibration subrange whose low temperature termination is at the $\mathrm{O}_{2} \mathrm{TP}$, equation 1 then becomes,

$$
W_{q}\left(T_{90}\right)=r_{q, 3}\left(T_{90}\right) r_{1, q}\left(T_{\text {tpw }}\right) r_{2,1} r_{3,2} .
$$

Also, following the example in the previous section, for temperatures of $39.4 \mathrm{~K}$ and below, equation 1 becomes

$$
W_{q}\left(T_{90}\right)=r_{q, 4}\left(T_{90}\right) r_{1, q}\left(T_{\text {tpw }}\right) r_{2,1} r_{3,2} r_{4,3} .
$$

Again, it may appear simpler from a mathematical point of view to substitute the single ratio measurement of $r_{4,2}(\approx 0.04)$ for the two measurements of $r_{3,2} r_{4,3}(\approx 0.4 \cdot 0.1)$. The ratio measurement of $r_{3,2}$, however, will always be necessary anyway for all calibration subranges extending below $83.8 \mathrm{~K}$. Also, a measurement of $r_{4,2}$ requires using higher currents than are customary in the use of the transfer standard at NIST. Furthermore, the resulting uncertainty in $W_{q}\left(T_{90}\right)$ from using the equation 6 as written above with five-products, is negligibly different from the uncertainty of its fourproduct reduction.

5.1.2 Calibrated-Standards Method: A general expression that applies to any ratio measurement system where all of the resistance standards are calibrated in ohms can be written as

$$
W_{q}\left(T_{90}\right)=r_{q j}\left(T_{90}\right) r_{1, q}\left(T_{\text {tpw }}\right) \frac{R_{j}}{R_{1}}
$$

where $R_{j}$ refers to any calibrated resistor $j=1,2, \ldots m$. In the special case where $j=1$, equation 7 reduces to equation 2 and the uncertainties in the calibration of the resistor do not contribute to the uncertainty in $W\left(T_{90}\right)$. However, in cases where $j \neq 1$, the uncertainties in the calibration of each of the two 
resistance standards do contribute to the uncertainty in $W\left(T_{90}\right)$ and to the overall calibration uncertainty of the SPRT.

At first glance, equation 7 may appear identical to equation 3 for the case of the ratio chain method. This may be true from the point of view of calculating $W\left(T_{90}\right)$, but we will see in section 6 that the two equations are treated differently when calculating the uncertainty in $W\left(T_{90}\right)$. The calibrated-standards method has the advantage that separated measurement systems can be independently operated while maintaining nominally equivalent resistance standards. Thus, equation 7 remains valid for the calculation of every $W\left(T_{90}\right)$ value and its associated uncertainty no matter how many different laboratories are involved in the calibration of the SPRT. However, all resistance standards must still be periodically recalibrated and they must be used at the same temperature as that at which they are calibrated.

\subsection{RIRTs}

At NIST, all RIRT calibrations are reported in ohms. Thus, the resistance at every calibration point is derived according to the simple relation,

$$
R_{q}\left(T_{90}\right)=r_{q, j}\left(T_{90}\right) R_{j}
$$

where $R_{j}$ is either a $10 \Omega$ or $100 \Omega$ standard resistor. The calibrated-standards method is used for all RIRT calibrations.

\section{Measurement Uncertainty}

The NIST assessment of uncertainties in the ITS-90 calibration of a thermometer involves the decomposition of uncertainty into Type A and Type B components. The Type A component $s$ is a combined uncertainty from standard deviations of only those measurements under direct statistical process control. The Type B components $u_{j}$ are the estimated standard uncertainties for each known component in the measurement process that cannot be directly measured $[7,8]$. In addition, uncertainties are described by a coverage factor $k$ which allows an estimated uncertainty of the measurements to be expanded to a specific level of confidence. The resulting expanded uncertainty, when quoted with a coverage factor of $k=2$, gives a level of confidence in the measurement of approximately $95 \%$ when the degrees of freedom $v \geq 50$, as is consistent with international practice.

The uncertainties reported here do not include any estimates for: 1) the uncertainties that may exist in the possible different realizations of the ITS -90 between national standards laboratories in other countries and NIST; 2) the non-uniqueness of the ITS-90;3) any effects that may be introduced by transportation of the thermometer between NIST and the user's laboratory; 4) long-term drift of the thermometer; and 5) any measurement uncertainties that exist in the user's laboratory. 


\subsection{SPRTs}

The general expression used for the calculation of uncertainties in $W\left(T_{90}\right)$ is given by,

$$
\left[u\left(W\left(T_{90}\right)\right)\right]^{2}=\sum_{j}\left(\frac{\partial W}{\partial x_{j}}\right)^{2}\left[u\left(x_{j}\right)\right]^{2}
$$

where $x_{j}$ is any independent quantity necessary to construct $W\left(T_{90}\right)$, either a measured resistance ratio or an absolute resistance. The $u\left(x_{\mathrm{j}}\right)$ are standard uncertainties, of either Type A or Type B.

\subsubsection{Type A Uncertainty}

For capsule SPRTs, the Type A standard uncertainty $s$ is determined from the standard deviations of all the individual ratio measurements that are necessary to construct each of the $W\left(T_{90}\right)$ values. For each calibration point and for each of the cases considered in section $5, s$ can be evaluated and expressed as an equivalent temperature uncertainty or $s=u_{\mathrm{A}}\left(W\left(T_{90}\right)\right) /(\mathrm{d} W / \mathrm{d} T)$. In the case of Type A uncertainties, the individual $x_{j}$ in equation 9 are the measured resistance ratios $r_{i, j}$. In the case of the ratio-chain method, a straightforward calculation using equation 1 and equation 9 leads to

$$
\left[u_{\mathrm{A}}\left(W_{q}\right)\right]^{2}=W_{q}^{2}\left[\left(\frac{u\left(r_{q, m}\left(T_{90}\right)\right)}{r_{q, m}\left(T_{90}\right)}\right)^{2}+\left(\frac{u\left(r_{1, q}\left(T_{\mathrm{tpw}}\right)\right)}{r_{1, q}\left(T_{\mathrm{tpw}}\right)}\right)^{2}+\sum_{j=2}^{m}\left(\frac{u\left(r_{j, j-1}\right)}{r_{j, j-1}}\right)^{2}\right]
$$

where $u_{\mathrm{A}}\left(W_{q}\right)$ represents the Type A uncertainty in the measured $W$ value for the $q$ th thermometer and where $u\left(r_{j, j-l}\right)$ represents the standard deviation of the measured ratio of resistance of standard $j$ to standard $j-1$.

In the case of the calibrated-standards method, the Type A uncertainty is derived from the thermometer ratio measurements alone, or

$$
\left[u_{\mathrm{A}}\left(W_{q}\right)\right]^{2}=W_{q}^{2}\left[\left(\frac{u\left(r_{q, m}\left(T_{90}\right)\right)}{r_{q, m}\left(T_{90}\right)}\right)^{2}+\left(\frac{u\left(r_{1, q}\left(T_{\mathrm{tpw}}\right)\right)}{r_{1, q}\left(T_{\mathrm{tpw}}\right)}\right)^{2}\right] .
$$

The uncertainties in the calibrations of the standard resistors must be included also, but they will be treated as a Type B component in the next section. 
The standard deviations of measured ratios $r_{q . m}$ for capsule SPRTs at the ITS-90 defining fixed points at or below the Ar TP are given in table III. The values of resistance ratios are based on the pooled variance of a set of 4 stable reference SPRTs maintained at NIST and measured concurrently in the comparator block during a single batch comparison in Laboratory $\mathrm{C}$. The reference SPRTs used here are all of nearly identical construction with $\mathrm{R}(273.16 \mathrm{~K}) \approx 25.5 \Omega$. The measurements were performed with an automatic balancing ac resistance ratio bridge having: a ratio resolution of $5 \times 10^{-9}$, a carrier frequency of $30 \mathrm{~Hz}$, a bandwidth of $0.1 \mathrm{~Hz}$, and measurement currents $i_{1}$ and $i_{2}$ as given. The statistical degrees of freedom are given under the heading " $\nu$ ".

Table III. Type A standard uncertainties for resistance ratio measurements of capsule SPRTs between $13.8 \mathrm{~K}$ and $83.8 \mathrm{~K}$.

\begin{tabular}{|l|c|c|c|c|c|c|}
\hline \hline$T_{90}(\mathrm{~K})$ & $r_{q, m}\left(T_{90}\right)$ & $R_{m}(\Omega)$ & $i_{1}, i_{2}(\mathrm{~mA})$ & $v$ & $u\left(r_{q, m}\right) / r_{q, m}$ & $s_{q}(\mathrm{mK})$ \\
\hline 83.8058 & 0.55 & 10 & $1.0,2.0$ & 380 & $2.24 \times 10^{-7}$ & 0.011 \\
\hline 54.35 & 0.234 & 10 & $1.0,2.0$ & 361 & $3.97 \times 10^{-7}$ & 0.009 \\
\hline 24.5561 & 0.22 & 1 & $2.0,2.828$ & 133 & $3.55 \times 10^{-6}$ & 0.025 \\
\hline 20.3 & 0.11 & 1 & $2.0,2.828$ & 95 & $4.28 \times 10^{-6}$ & 0.025 \\
\hline 17.0 & 0.06 & 1 & $2.828,5.0$ & 76 & $3.95 \times 10^{-6}$ & 0.021 \\
\hline 13.8033 & 0.03 & 1 & $2.828,5.0$ & 190 & $6.38 \times 10^{-6}$ & 0.036 \\
\hline
\end{tabular}

The values of $u\left(r_{q, m}\right) / r_{q . m}$ as shown in Table III are primarily limited by the SPRT signal-to-noise ratio of the measurement system in Lab C. The equivalent uncertainty in $\mathrm{mK}$ is shown under $s_{q}$ which represents the contribution to the Type A uncertainty from the SPRT comparison measurement only. The other components of $u_{\mathrm{A}}\left(W_{q}\left(T_{90}\right)\right)$, arising from $u\left(r_{1, q}\right)$ and $u\left(r_{j, j-1}\right)$ in equation 10, are significantly smaller than $u\left(r_{q, m}\right)$ which results in $s_{q}$ being the dominant contribution to the type A uncertainty. Under ideal conditions, the statistics associated with ratio measurements of $r_{j, j-1}$ are least count limited (i.e. $\left.u\left(r_{j, j-1}\right) \sim 5 \times 10^{-9}\right)$. This is true also in most cases for ratios $r_{1 . q}\left(T_{\text {tpw }}\right)$ with practically any SPRT in a properly prepared $\mathrm{H}_{2} \mathrm{O}$ triple-point cell. Thus, these additional components to $u_{\mathrm{A}}\left(W_{q}\left(T_{90}\right)\right)$ have a negligible effect when added in quadrature with the $u\left(r_{q, m}\right)$ values. Consequently, there is no practical difference between the various methodologies discussed in section 5 as far as type A uncertainties are concerned. Even when the extra measurements needed to complete the ratio chain between two or more separate measurement systems are included, the net effect on the calculated $u_{\mathrm{A}}\left(W_{q}\left(T_{90}\right)\right)$ value is negligible.

The overall Type A uncertainties $s$ for the various SPRT calibration points are given in table IV. The values of $W_{\mathrm{r}}$ and $\mathrm{d} W_{\mathrm{r}} \mathrm{d} T$ are computed from the SPRT reference function [4]. The Type A 
uncertainties are derived directly from equation 10 and the relation $s=u_{\Lambda}\left(W_{q}\left(T_{90}\right)\right) /\left(\mathrm{d} W_{\mathrm{r}} / \mathrm{d} T\right)$. The values for $u_{\Lambda}\left(W_{q}\left(T_{90}\right)\right)$ and $s$ apply equally well to either the ratio-chain method or the calibrated-standards method for constructing measured $W_{q}\left(T_{90}\right)$ values.

Table IV. Type A uncertainties for comparison calibrations of capsule SPRTs.

\begin{tabular}{|l|c|c|c|c|}
\hline \multicolumn{1}{|c|}{$T(\mathrm{~K})$} & $W_{\mathrm{r}}\left(T_{90}\right)$ & $\mathrm{d} W_{\mathrm{r}} \mathrm{d} T$ & $u_{\Lambda}\left(W_{q}\left(T_{90}\right)\right)$ & $s(\mathrm{mK})$ \\
\hline \hline 83.8058 & 0.2158597 & 0.0043416 & $2.45 \times 10^{-7}$ & 0.012 \\
\hline 54.3584 & 0.0917180 & 0.0039029 & $4.50 \times 10^{-7}$ & 0.011 \\
\hline 24.5561 & 0.0084497 & 0.0012267 & $3.68 \times 10^{-6}$ & 0.025 \\
\hline 20.3 & 0.0042580 & 0.0007523 & $4.52 \times 10^{-6}$ & 0.026 \\
\hline 17.0 & 0.0022805 & 0.0004581 & $4.39 \times 10^{-6}$ & 0.022 \\
\hline 13.8033 & 0.0011901 & 0.0002406 & $7.31 \times 10^{-6}$ & 0.036 \\
\hline
\end{tabular}

6.1.2 Type B uncertainty. The Type B uncertainties for capsule SPRT calibrations must take into account the limitations in accuracy of the resistance ratio measurements, as well as the uncertainties in the fixed point realizations and the comparison process. In addition, there are complications from the subtle differences in the methodologies described in section 5 . Table $\mathrm{V}$ lists values for the individual Type B components, $u_{j}$, described below. The combined Type B uncertainty is given by

$$
u_{\mathrm{B}}=\sqrt{\sum_{j} u_{j}^{2}}
$$

6.1.2.1 Bridge Accuracy, $u_{1}$. The accuracy in the measured ac resistance ratio is generally limited by non-linearities in the inductive characteristics of the ratio transformer. In principle, these non-linearities can be measured, corrections evaluated, and applied to effectively calibrate the bridge [22]. However, this is not practical for the type of commercial bridges used at NIST at the present time. Currently, bridge performance is simply "verified" by using either a "ratio test set", as supplied by the bridge manufacturer [23], or by performing a series of three-way substitution tests [24]. Both methods are capable only of verifying the overall accuracy specification for a bridge. The commercial ac resistance bridges currently used at NIST are specified by the manufacturer as accurate to within " $\pm 2 \times 10^{-7}$ ratio" $|23|$. Periodic bridge verification tests conducted at NIST have been consistent with this figure. Hence, we model the uncertainty in measured ratios associated with the bridge non-linearity as a rectangular distribution with a width of $2 a=4 \times 10^{-7}$. To this we add an additional uncertainty to reflect the least count contribution as another rectangular distribution of 
width $2 b=5 \times 10^{-9} / r$. Thus, our estimated Type B standard uncertainty (squared) for the bridge accuracy becomes,

$$
u_{1}^{2}=\left(\frac{W\left(T_{90}\right)}{\frac{d W}{d T}}\right)^{2} \frac{1}{3} \sum_{i}\left(a^{2}+b^{2}\right)_{i}
$$

where the summation is over the number of independent ratio measurements made in the process of constructing $W\left(T_{90}\right)$. For simplicity in the remaining discussion we asssume $W\left(T_{90}\right)=W_{q}\left(T_{90}\right)$.

A four-terminal resistor network being developed at the Measurement Standards Laboratory of New Zealand has recently been shown to facilitate a practical calibration scheme for resistance ratio bridges [25]. By employing such a network calibrator to ac resistance bridges used at NIST, it should be possible to reduce the uncertainty component $u_{1}$ by a factor of 10 to 20 . However, for calibrations at $83.8 \mathrm{~K}$ and below, these uncertainties are already practically insignificant.

6.1.2.2 Standard Resistors, $u_{2}$. When using the calibrated-standards method, the accuracy of the calibrations of the individual standards used is treated as a Type B uncertainty component. Essentially, the "ohm" as represented by a $10 \Omega$ standard may not be exactly equivalent to the "ohm" as represented by a $100 \Omega$ standard, which leads to an error in constructing the $W\left(T_{90}\right)$ for some SPRT subranges. At NIST, those resistance standards which are calibrated in ohms for use within the Thermometry Group are calibrated by the Electricity Division according to the procedures given in Technical Note 1298 [26]. The relative expanded $(k=2)$ uncertainty of these calibrations is usually $U_{\mathrm{R}}=0.5 \times 10^{-6}$ to $1.0 \times 10^{-6}$ for the decade resistances of $1 \Omega, 10 \Omega$, and $100 \Omega$. According to equations 7 and 10 , the uncertainty $U_{\mathrm{R}}$ will contribute twice, once for each of the two different standards used in the construction of $W\left(T_{90}\right)$. Thus, we assign a normal distribution to this component with a standard uncertainty,

$$
u_{2}=\frac{1}{2}\left(\frac{W\left(T_{90}\right)}{\frac{d W}{d T}}\right) U_{R} \quad \text { (Calibrated-Standards Method) }
$$

In the case of the ratio-chain method, however, the calibration uncertainties of the resistance standards are irrelevant, and thus $u_{2}=0$.

6.1.2.3 Fixed-Point Realizations, $u_{3}$. This category includes all of the sources of uncertainty involved in performing the realizations of the required fixed points. These uncertainties are discussed in detail elsewhere [27]. 
6.1.2.4 Reference SPRT Calibration, $u_{4}$. Apart from the uncertainties of the fixed-point realizations, a reference SPRT must be calibrated as well by constructing $W\left(T_{90}\right)$ values and then repeatedly measured during a batch calibration process in order to assign a temperature to the comparator block. As such, the reference is subject to many of the same uncertainties as any other SPRT being calibrated. Thus, the reference SPRT adds uncertainty components to the process that are equivalent to $s, u_{1}$, and possibly $u_{2}$, depending on how the measurements are made. To account for this additional uncertainty, we assign a Type B component $u_{4}=\left(s^{2}+u_{1}{ }^{2}+u_{2}{ }^{2}\right)^{1 / 2}$.

6.1.2.5 Comparisons, $u_{5}$. In making comparison measurements, there is always the possibility that the reference SPRT is not in perfect thermal equilibrium with the thermometer being calibrated. Also, if the comparison block temperature is changing with time, thermal gradients will exist. In some cases, gradients can exist even in steady state conditions. The standard practice for detecting these conditions is to include one or two check thermometers in the batch calibration that carry an equivalent calibration to the reference thermometer. If the observed difference in temperature between the reference and check thermometers at a fixed point temperature is statistically significant, and otherwise unpreventable, then some allowance $u_{5}$ must be made to account for this in the uncertainty.

Normally, with close fitting thermometer wells, an oxygen-free copper comparator block under high vacuum conditions should support only negligible thermal gradients. However, any such disagreement $\delta T$ that is observed in a batch calibration process, and that can not be further minimized, must be evaluated in comparison to all other known sources of uncertainty first. If $U_{\mathrm{T}}$ is the expanded uncertainty (see section 6.1 .3 below) for the calibration process, then $u_{5}$ is estimated by adjusting its value so that $U_{\mathrm{T}} \leq \delta T$ at all calibration point temperatures. In addition, $u_{5}$ is constrained to be a slowly varying, monotonic, increasing function of temperature. This last feature is physically reasonable for any model of thermal gradients in the copper block.

Table V. Type B uncertainty components for SPRT comparison calibrations, in $\mathrm{mK}$. The total Type B uncertainty is shown for the ratio-chain method $u_{\mathrm{Brcm}}$ and calibrated-standards method, $u_{\mathrm{Bcss}}$.

\begin{tabular}{|c|c|c|c|c|c|c|c|}
\hline$T(\mathrm{~K})$ & $\begin{array}{c}u_{1} \\
\text { RCM, CSM }\end{array}$ & $\begin{array}{c}u_{2} \\
\text { RCM, CSM }\end{array}$ & $u_{3}$ & $u_{4}$ & $u_{5}$ & $u_{\text {Brcm }}$ & $u_{\text {Bcsm }}$ \\
\hline \hline 83.8058 & $0.012,0.008$ & $0,0.028$ & 0.06 & 0.031 & 0.10 & 0.121 & 0.124 \\
\hline 54.3584 & $0.005,0.004$ & $0,0.013$ & 0.03 & 0.017 & 0.08 & 0.087 & 0.088 \\
\hline 24.5561 & $0.002,0.001$ & $0,0.004$ & 0.11 & 0.026 & 0.07 & 0.129 & 0.129 \\
\hline 20.3 & $0.002,0.001$ & $0,0.003$ & 0.08 & 0.026 & 0.065 & 0.106 & 0.106 \\
\hline 17.0 & $0.001,0.001$ & $0,0.003$ & 0.08 & 0.022 & 0.063 & 0.104 & 0.104 \\
\hline 13.8033 & $0.002,0.001$ & $0,0.003$ & 0.075 & 0.036 & 0.060 & 0.103 & 0.103 \\
\hline
\end{tabular}


Of all the Type B components for SPRT calibrations, $u_{5}$ is currently the dominant contribution at comparison temperatures of approximately $50 \mathrm{~K}$ and higher. At lower comparison temperatures, both $u_{5}$ and $u_{3}$ dominate. Even though the RCM and CSM values for $u_{1}$ and $u_{2}$ are significantly different for most of the comparison temperatures, both are generally negligible in comparison to $u_{5}$ and $u_{3}$. Consequently, the difference between $u_{\mathrm{Brcm}}$ and $u_{\mathrm{Bcsm}}$ is negligible for all temperatures.

6.1.3 Total Calibration Uncertainty for SPRTs. The uncertainties described so far must be combined and expanded in a uniform way at each ITS-90 calibration point. These combined expanded uncertainties then provide a means for calculating the propagated uncertainty throughout each of the defined subranges.

6.1.3.1 Expanded Uncertainty. The Type A and Type B uncertainties at each of the SPRT calibration points are combined in quadrature and expanded by the coverage factor $k$ to form the expanded uncertainty given by

$$
U_{\mathrm{T}}=k \sqrt{s^{2}+u_{\mathrm{B}}^{2}} .
$$

NIST expanded uncertainties are quoted with a coverage factor of $k=2$ unless stated otherwise. Table Vl lists the values of $U_{\mathrm{T}}$ according to equation 15 for all of the ITS- 90 calibration points relevant to capsule SPRTs. The values quoted from the Ar TP and higher are the latest estimates of uncertainty incorporating the most recent fixed-point materials now in use at NIST.

Table VI. NIST expanded uncertainties for capsule SPRT calibrations.

\begin{tabular}{|c|c|c|}
\hline Calibration Point & $T_{90}(\mathrm{~K})$ & Expanded Uncertainty, $U_{\mathrm{T}}(\mathrm{mK})$ \\
\hline $\mathrm{e}-\mathrm{H}_{2} \mathrm{TP}^{\dagger}$ & 13.8033 & 0.22 \\
\hline $\mathrm{e}-\mathrm{H}_{2} \mathrm{VP}^{\dagger}$ & 17.0 & 0.21 \\
\hline $\mathrm{e}-\mathrm{H}_{2} \mathrm{VP}^{+}$ & 20.3 & 0.22 \\
\hline $\mathrm{Ne} \mathrm{TP}^{\dagger}$ & 24.5561 & 0.26 \\
\hline $\mathrm{O}_{2} \mathrm{TP}^{\dagger}$ & 54.3584 & 0.18 \\
\hline $\mathrm{ArTP}^{+}$ & 83.8058 & 0.25 \\
\hline $\mathrm{Ar} \mathrm{TP}$ & 83.8058 & 0.12 \\
\hline $\mathrm{Hg} \mathrm{TP}$ & 234.3156 & 0.20 \\
\hline $\mathrm{H}_{2} \mathrm{O}$ TP & 273.16 & 0.04 \\
\hline Ga MP & 302.9146 & 0.04 \\
\hline In FP & 429.7485 & 0.32 \\
\hline Sn FP & 505.078 & 0.30 \\
\hline
\end{tabular}


6.1.3.2 Total Calibration Uncertainty. The total calibration uncertainty at any temperature within a given temperature subrange is determined from the expanded uncertainties propagated from each of the relevant defining fixed points. The uncertainty propagated from each defining fixed point is calculated by assuming the appropriate uncertainty at that fixed point but with no uncertainty at the other fixed points, and numerically determining how that uncertainty propagates. The total uncertainty from a calibration is then determined in part by calculating the root-sum-square (RSS) uncertainty arising from all of the defining fixed points used in that calibration. Table VII gives estimates of the maximum uncertainty of calibrations for each temperature subrange for capsule SPRTs calibrated at NIST. Also shown in this table is the approximate temperature where this maximum in the propagated uncertainty occurs. Figures 1-8 show uncertainty propagation curves for the ITS-90 temperature subranges discussed here, using the uncertainties of the fixed points given in Table VI. The thick line represents the RSS uncertainty for the subranges based on those uncertainties.

The propagated-uncertainty curve for the triple-point-of-water (TPW), as shown in Figure 9 (an example which assumes $0.1 \mathrm{mK}$ uncertainty at the TPW), is the uncertainty incurred by the user, not an uncertainty in the NIST calibration. During the NIST calibration of a thermometer, any uncertainty from a measurement at the TPW is incorporated into the definition of $W\left(T_{90}\right)$. If the uncertainty made by the user at the TPW is not $0.1 \mathrm{mK}$, a corrected propagated uncertainty at any temperature can be calculated by using the appropriate multiplicative factor.

Table VII. Estimates of Maximum Uncertainties of Calibrations for capsule SPRTs at NIST.

\begin{tabular}{ccccc}
\hline \hline \multicolumn{2}{c}{ Temperature Subrange } & Maximum Uncertainty (RSS), mK & $T_{\max }(\mathrm{K})$ \\
\hline $13.8033 \mathrm{~K}$ & to $273.16 \mathrm{~K}$ & 0.62 & 15 \\
$24.5561 \mathrm{~K}$ & to $273.16 \mathrm{~K}$ & 0.40 & 33 \\
$54.8058 \mathrm{~K}$ & to $273.16 \mathrm{~K}$ & 0.29 & 183 \\
$83.8058 \mathrm{~K}$ & to $273.16 \mathrm{~K}$ & 0.39 & 159 \\
$234.3156 \mathrm{~K}$ & to $302.9146 \mathrm{~K}$ & 0.20 & 234 \\
$273.15 \mathrm{~K}$ & to $302.9146 \mathrm{~K}$ & 0.04 & 303 \\
$273.15 \mathrm{~K}$ & to $429.7485 \mathrm{~K}$ & 0.32 & 429 \\
$273.15 \mathrm{~K}$ & to $505.078 \mathrm{~K}$ & 0.37 & 385 \\
\hline \hline
\end{tabular}

\subsection{RIRTs.}

In the case of RIRTs, calibrations are reported directly in ohms and their standard uncertainty represents the standard deviation of $R\left(T_{90}\right)$. Ratio measurements are treated as Type $\mathrm{A}$ uncertainties while resistance calibrations are treated as Type B uncertainties. As in the case of SPRTs, a general equation similar to equation 9 for calculating the various components in the combined uncertainty can be written as 


$$
\left[u\left(R\left(T_{90}\right)\right)\right]^{2}=\sum_{j}\left(\frac{\partial R}{\partial x_{j}}\right)^{2}\left[u\left(x_{j}\right)\right]^{2} .
$$

6.2.1 Type A uncertainty. The Type A standard uncertainties for ratio measurements of RIRTs are shown in Table VIII. The RIRT calibration ranges are sub-divided into 19 temperature subranges. These RIRT subranges are not ITS-90 defined, rather they are simply an expedient means of treating the statistics, given the interpolating properties of RIRTs. The values shown for $R$ and $\mathrm{d} R / \mathrm{d} T$ are typical of a $100 \Omega\left(T_{90}=273.15 \mathrm{~K}\right)$ RIRT. The values of $s$ are based on the pooled variance from two similar reference RIRTs below $13.8 \mathrm{~K}$ and three reference RIRTs above $13.8 \mathrm{~K}$, all of which are maintained at NIST. Measurements were taken concurrently in the comparator block during a single batch comparison in Laboratory $\mathrm{C}$. The measurements were performed with an automatic balancing ac resistance ratio bridge having a ratio resolution of $5 \times 10^{-9}$, a carrier frequency of $30 \mathrm{~Hz}$, a bandwidth $0.1 \mathrm{~Hz}$, and measurement currents $i_{1}$ and $i_{2}$ as given. The statistical degrees of freedom are given under the heading " $\nu$ ".

6.2.2 Type B Uncertainty. The Type B uncertainties for capsule RIRT calibrations take into account the limitations in accuracy for the resistance ratio measurements, the uncertainties in the fixed-point realizations, and the comparison process in a similar manner to the calibrated-standards method for SPRTs. Equation 12 is used for calculating the total Type B standard uncertainty. For RIRTs, there are some additional complications in the range between $13.8033 \mathrm{~K}$ and $24.5561 \mathrm{~K}$ due to the overlapping definitions of the ITS-90. The values given here are valid for capsule RIRTs with a nominal resistance of $100 \Omega$ at $273.15 \mathrm{~K}$ and a nominal composition (atomic fraction: $0.5 \% \mathrm{Fe}, 99.5 \%$ $\mathrm{Rh})$.

6.2.2.1 Bridge Accuracy, $u_{1}$. This contribution is treated in exactly the same manner as in section 6.1.2.1. As before, we model the uncertainty in measured ratios associated with the bridge nonlinearity as two rectangular distributions, one with a width of $2 a=4 \times 10^{-7}$ and another of width $2 b=5 \times 10^{-9} / r$. Equation 13 is used to calculate $u_{1}$ using these same values. As in the case of SPRTs, this uncertainty component is practically insignificant, having a maximum value of only $0.01 \mathrm{mK}$ over the range of RIRT calibration temperatures.

6.2.2.2 Standard Resistors, $u_{2}$. For the type of ac resistance bridge used at NIST, a $100 \Omega$ resistance standard is generally used when measuring RIRTs at $13.8 \mathrm{~K}$ and above. For temperatures below $13.8 \mathrm{~K}$, a $10 \Omega$ standard may be used. The choice of $10 \Omega$ or $100 \Omega$ for temperatures below $13.8 \mathrm{~K}$, however, is not critical for maintaining adequate resolution. This is due to the fact that the RIRT sensitivity, unlike that of the SPRT, increases with decreasing temperature reaching a value as high as $10 \% \mathrm{~K}^{-1}$ at $0.65 \mathrm{~K}$. For this reason, RIRT calibrations rely almost entirely on a $100 \Omega$ standard throughout the range of calibration. In the range between $3.4 \mathrm{~K}$ and $12.0 \mathrm{~K}$, a $10 \Omega$ standard may 
Table VIII. Type A standard uncertainties for comparison measurements of RIRTs made between $0.65 \mathrm{~K}$ and $90.0 \mathrm{~K}$.

\begin{tabular}{|l|c|c|c|c|c|c|}
\hline \hline Range $(\mathrm{K})$ & $R_{\max }(\Omega)$ & $R_{\mathrm{s}}(\Omega)$ & $i_{1}, i_{2}(\mathrm{~mA})$ & $\mathrm{d} R / \mathrm{d} T_{\max }$ & $v$ & $s(\mathrm{mK})$ \\
\hline 0.65 to 0.8 & 6.84 & 100 & $0.141,0.2$ & 0.72 & 57 & 0.011 \\
\hline 0.8 to 1.1 & 7.05 & 100 & $0.141,0.2$ & 0.69 & 76 & 0.014 \\
\hline 1.1 to 1.5 & 7.32 & 100 & $0.2,0.282$ & 0.66 & 76 & 0.010 \\
\hline 1.5 to 2.0 & 7.64 & 100 & $0.2,0.282$ & 0.62 & 76 & 0.010 \\
\hline 2.0 to 2.7 & 8.06 & 100 & $0.2,0.282$ & 0.58 & 76 & 0.009 \\
\hline 2.7 to 3.4 & 8.45 & 100 & $0.2,0.282$ & 0.53 & 76 & 0.009 \\
\hline 3.4 to 4.7 & 9.10 & 10 & $0.2,0.282$ & 0.46 & 133 & 0.013 \\
\hline 4.7 to 6.3 & 9.78 & 10 & $0.2,0.282$ & 0.40 & 76 & 0.012 \\
\hline 6.3 to 8.5 & 10.57 & 10 & $0.2,0.282$ & 0.33 & 76 & 0.014 \\
\hline 8.5 to 11.5 & 11.44 & 10 & $0.2,0.282$ & 0.26 & 57 & 0.019 \\
\hline 11.5 to 14.5 & 12.15 & 100 & $0.2,0.282$ & 0.21 & 247 & 0.016 \\
\hline 14.5 to 18.0 & 12.83 & 100 & $0.2,0.282$ & 0.18 & 247 & 0.014 \\
\hline 18.0 to 22.0 & 13.49 & 100 & $0.2,0.282$ & 0.15 & 323 & 0.024 \\
\hline 22.0 to 25.0 & 13.94 & 100 & $0.2,0.282$ & 0.145 & 342 & 0.027 \\
\hline 25.0 to 30.0 & 14.66 & 100 & $0.2,0.282$ & 0.145 & 285 & 0.024 \\
\hline 30.0 to 41.0 & 16.44 & 100 & $0.2,0.282$ & 0.185 & 114 & 0.018 \\
\hline 41.0 to 55.0 & 19.56 & 100 & $0.2,0.282$ & 0.26 & 114 & 0.011 \\
\hline 55.0 to 69.0 & 23.67 & 100 & $0.2,0.282$ & 0.32 & 209 & 0.012 \\
\hline 69.0 to 90.0 & 31.06 & 100 & $0.2,0.282$ & 0.37 & 190 & 0.014 \\
\hline
\end{tabular}

yield marginally better statistics than a $100 \Omega$ standard. At temperatures much lower than $3.4 \mathrm{~K}$, however, the RIRT sensitivity is so large that it is necessary to decrease the bridge gain when using a $10 \Omega$ standard. The standards used in this assessment are shown in the third column of table VIII. Here again, we use standard calibration uncertainties of

$$
u_{2}=\frac{1}{2}\left(\frac{R\left(T_{90}\right)}{\frac{\mathrm{d} R}{\mathrm{~d} T}}\right) U_{R}
$$


where $U_{\mathrm{R}}=1.0 \times 10^{-6}$ for $10 \Omega$ standards and $U_{\mathrm{R}}=0.5 \times 10^{-6}$ for $100 \Omega$ standards when they are calibrated at $25^{\circ} \mathrm{C}$ according to the procedures given in Technical Note 1298 [24].

6.2.2.3 Realization, $u_{3}$. There are five distinct subranges in the NIST realization of the ITS-90 that underlie the RIRT calibration ranges discussed here.

i) $0.65 \mathrm{~K}$ to $2.0 \mathrm{~K}$. This subrange is based on the ITS- $90{ }^{3} \mathrm{He}$ vapor pressure relations. The uncertainties are derived from the pressure measurements [18].

ii) $2.0 \mathrm{~K}$ to $5.0 \mathrm{~K}$. This subrange is based on the ITS- $90{ }^{4} \mathrm{He}$ vapor pressure relations. The uncertainties are derived from the pressure measurements [18].

iii) $5.0 \mathrm{~K}$ to $13.8 \mathrm{~K}$. This subrange is based on the ITS-90 defined interpolating constant volume gas thermometer (ICVGT). The uncertainties are derived from the pressure measurements, pressure corrections, and fixed point realizations according to the ITS-90 definition [19].

iv) $13.8 \mathrm{~K}$ to $24.5561 \mathrm{~K}$. This subrange is based on the ITS-90 defined reference function and deviation function for SPRTs over the range $13.8 \mathrm{~K}$ to $273.16 \mathrm{~K}$. The uncertainties in the defining fixed points are propagated to calculate estimated uncertainties between the fixed point temperatures as described in section 6.1.2.3. Alternatively, a reference RIRT may be used to transfer the ITS-90 ICVGT scale definition [19].

v) $24.5561 \mathrm{~K}$ to $83.8 \mathrm{~K}$. This subrange is based on the ITS-90 defined reference function and deviation function for SPRTs over the range $13.8 \mathrm{~K}$ to $273.16 \mathrm{~K}$. The uncertainties in the defining fixed points are propagated to calculate estimated uncertainties between the fixed points as described in section 6.1.2.3.

6.2.2.4 Reference Calibration, $u_{4}$. The uncertainty in the calibration of the reference thermometer is analogous to the case treated above for SPRTs. This component allows for a Type A measurement uncertainty for the reference as well as several Type B uncertainties that must be associated with the measurement of the reference, all of which are added in quadrature to arrive at an estimate for $u_{4}$. However, there are three distinct temperature subranges that differ in the type of reference thermometer appropriate for the calibration.

i) $0.65 \mathrm{~K}$ to $13.8 \mathrm{~K}$. The reference thermometer is an RIRT calibrated on the NIST He vapor pressure scales up to $5.0 \mathrm{~K}$ and the ICVGT scale from $5.0 \mathrm{~K}$ to $13.8 \mathrm{~K}$. During comparison calibrations of RIRTs, the reference RIRT is measured at a finite reference current to determine the temperature of the comparison block. Under these conditions, at temperatures much below $5 \mathrm{~K}$, the dominant contribution to $u_{4}$ is due to the relatively large and rapidly varying (with temperature) self-heating effects exhibited by a capsule RIRT as described under "self-heating" below.

ii) $13.8 \mathrm{~K}$ to $24.5561 \mathrm{~K}$. In this sub-range, a RIRT calibration will normally switch over to an SPRT reference thermometer. Hence, in this case the value assigned to $u_{4}$ will be equivalent to that described in section 6.1.2.4. In addition, reference RIRTs are maintained at NIST which carry the ICVGT 
version of ITS-90 up to $24.5561 \mathrm{~K}$. Table X lists Type B uncertainties for both of these cases for comparison. However, calibrations of RIRTs in this sub-range in terms of the ICVGT realization at NIST are performed only when a special request is made by the customer. Otherwise, the SPRT definition of the ITS-90 will be disseminated to customers using RIRTs above $13.8 \mathrm{~K}$.

iii) $24.5561 \mathrm{~K}$ to $90 \mathrm{~K}$. In this sub-range the RIRT is calibrated exclusively against a reference SPRT and the value assigned to $u_{4}$ will be equivalent to that described in section 6.1.2.4.

6.2.2.5 Comparison, $u_{5}$. In the range $13.8 \mathrm{~K}$ to $83.8 \mathrm{~K}$, this uncertainty component is exactly the same as that estimated for the case of SPRTs. The reason being that $u_{5}$ represents the uncertainty due to properties of the comparison block and the flux of heat into and out of that block, independent of the particular type of thermometers mounted within it. For temperatures below $13.8 \mathrm{~K}$, an estimate of the temperature gradients is extrapolated downward with values that are consistent with data from reference RIRT comparisons. There are no estimates made for $u_{5}$ below $4.2 \mathrm{~K}\left(u_{5}=0.01 \mathrm{mK}\right)$ due to the fact that any such gradients would be too small to be measured.

6.2.2.6 Self Heating, $u_{6}$. These effects are most noticeable in RIRTs at temperatures below $4 \mathrm{~K}$. In this case, they are due to the strong changes in the thermal transport properties of the ${ }^{4} \mathrm{He}$ fill gas with decreasing temperature as the gas condenses and undergoes a superfluid transition under unsaturated conditions [11]. The standard uncertainty assigned for this self-heating effect is $10 \%$ of the size of the effect at the normal calibration current $(i=0.2 \mathrm{~mA}$ for $T<1.0 \mathrm{~K}$ and $i=0.282 \mathrm{~mA}$ for $T>1.0 \mathrm{~K})$ which often reaches a peak value of approximately $0.3 \mathrm{mK}$ at $T_{90}=1.6 \mathrm{~K}$ for $i=0.282 \mathrm{~mA}$.

The underlying assumption here is that the magnitude of the self-heating is probably not perfectly reproducible under finite current conditions. In addition, the detailed characteristics of RIRT self heating below $5 \mathrm{~K}$ can vary considerably from one device to the next. However, this uncertainty component can be eliminated by measuring the resistance of each device at two currents and extrapolating to zero power dissipation. NIST RIRT calibrations are provided with two current data, however, a user may not necessarily use the RIRT is this way, in which case the component $u_{6}$ should be added as a Type B uncertainty. Estimates for $u_{6}$ are not given above $5 \mathrm{~K}$, where the self-heating effects in RIRTs are generally smaller in size and exhibit only a very weak temperature dependence.

6.2.3. Expanded Uncertainty for RIRTs. The expanded uncertainty for RIRT calibrations is calculated according to equation 15. The Type B uncertainties for the ICVGT defined scale and the SPRT defined scale are treated separately in the range between $13.8 \mathrm{~K}$ and $24.5561 \mathrm{~K}$. Hence, the expanded uncertainty is also separated in this range, as shown in Table XII. The Type B contribution to the combined uncertainty predominates over all RIRT calibration temperatures. In the region of overlapping definitions between $13.8033 \mathrm{~K}$ and $24.5561 \mathrm{~K}$, the SPRT definition has slightly lower uncertainties than the ICVGT definition using an RIRT reference at temperatures of $17 \mathrm{~K}$ and above. However, the ICVGT/RIRT definition is sightly less uncertain below $17 \mathrm{~K}$ due to the relatively large interpolation uncertainties associated with the SPRT in the region near $15 \mathrm{~K}$.

Figure 10 is a plot of the residual values (expressed in $\mathrm{mK}$ ) of a set of polynomial functions from three least-squares fits compared to the actual calibration data for a typical $100 \Omega$ RIRT. The expanded 
uncertainties $(k=2)$ at each calibration point are shown as uncertainty bars centered on each point. The fits were made in the same manner as described in section 2.2.

Table IX. Type B standard uncertainties for comparison measurements of RIRTs between $0.65 \mathrm{~K}$ and $13.8 \mathrm{~K}$.

\begin{tabular}{|c|c|c|c|c|c|c|}
\hline$T / \mathrm{K}$ & $u_{1} / \mathrm{mK}$ & $u_{2} / \mathrm{mK}$ & $u_{3} / \mathrm{mK}$ & $u_{4} / \mathrm{mK}$ & $u_{5} / \mathrm{mK}$ & $u_{6} / \mathrm{mK}$ \\
\hline \hline 0.65 & 0.001 & 0.002 & 0.07 & 0.023 & & 0.02 \\
\hline 0.85 & 0.001 & 0.002 & 0.06 & 0.019 & & 0.013 \\
\hline 1.18 & 0.001 & 0.002 & 0.06 & 0.011 & & 0.001 \\
\hline 1.64 & 0.001 & 0.002 & 0.06 & 0.30 & & 0.028 \\
\hline 2.2 & 0.001 & 0.002 & 0.023 & 0.024 & & 0.022 \\
\hline 2.8 & 0.001 & 0.003 & 0.023 & 0.016 & & 0.013 \\
\hline 3.414 & 0.001 & 0.006 & 0.023 & 0.025 & & 0.02 \\
\hline 4.2 & 0.002 & 0.007 & 0.023 & 0.021 & 0.010 & 0.014 \\
\hline 5.1 & 0.002 & 0.008 & 0.065 & 0.018 & 0.015 & 0.01 \\
\hline 6.1 & 0.002 & 0.009 & 0.067 & 0.016 & 0.020 & \\
\hline 7.2 & 0.002 & 0.011 & 0.069 & 0.018 & 0.026 & \\
\hline 8.6 & 0.003 & 0.013 & 0.071 & 0.031 & 0.033 & \\
\hline 10.2 & 0.004 & 0.015 & 0.074 & 0.025 & 0.041 & \\
\hline 12.0 & 0.004 & 0.018 & 0.077 & 0.020 & 0.051 & \\
\hline 13.8 & 0.005 & 0.011 & 0.080 & 0.020 & 0.060 & \\
\hline
\end{tabular}


Table X. Type B standard uncertainties for comparison measurements of RIRTs between $13.8 \mathrm{~K}$ and $24.5561 \mathrm{~K}$.

\begin{tabular}{|c|c|c|c|c|c|c|c|}
\hline & & & \multicolumn{2}{|c|}{ ICVGT/RIRT } & \multicolumn{2}{|c|}{ SPRT } & \\
\hline$T / \mathrm{K}$ & $u_{1} / \mathrm{mK}$ & $u_{2} / \mathrm{mK}$ & $u_{3} / \mathrm{mK}$ & $u_{4} / \mathrm{mK}$ & $u_{3} / \mathrm{mK}$ & $u_{4} / \mathrm{mK}$ & $u_{5} / \mathrm{mK}$ \\
\hline \hline 13.8 & 0.005 & 0.011 & 0.080 & 0.020 & 0.075 & 0.036 & 0.06 \\
\hline 14.6 & 0.006 & 0.12 & 0.083 & 0.064 & 0.221 & 0.033 & 0.061 \\
\hline 15.4 & 0.006 & 0.012 & 0.086 & 0.047 & 0.212 & 0.030 & 0.062 \\
\hline 17.036 & 0.007 & 0.014 & 0.092 & 0.031 & 0.080 & 0.022 & 0.062 \\
\hline 18.66 & 0.007 & 0.015 & 0.098 & 0.054 & 0.086 & 0.018 & 0.063 \\
\hline 20.27 & 0.008 & 0.017 & 0.104 & 0.128 & 0.080 & 0.025 & 0.065 \\
\hline 21.5 & 0.008 & 0.018 & 0.109 & 0.045 & 0.071 & 0.025 & 0.067 \\
\hline 22.6 & 0.009 & 0.019 & 0.113 & 0.054 & 0.076 & 0.025 & 0.068 \\
\hline 23.6 & 0.009 & 0.019 & 0.116 & 0.054 & 0.091 & 0.025 & 0.069 \\
\hline 24.556 & 0.009 & 0.20 & 0.120 & 0.036 & 0.105 & 0.025 & 0.070 \\
\hline
\end{tabular}

Table XI. Type B standard uncertainties for comparison measurements of RIRTs between $24.5561 \mathrm{~K}$ and $83.8 \mathrm{~K}$.

\begin{tabular}{|c|c|c|c|c|c|}
\hline$T / \mathrm{K}$ & $u_{1} / \mathrm{mK}$ & $u_{2} / \mathrm{mK}$ & $u_{3} / \mathrm{mK}$ & $u_{4} / \mathrm{mK}$ & $u_{5} / \mathrm{mK}$ \\
\hline \hline 26.0 & 0.010 & 0.021 & 0.123 & 0.023 & 0.070 \\
\hline 27.1 & 0.010 & 0.021 & 0.130 & 0.021 & 0.071 \\
\hline 31.2 & 0.010 & 0.021 & 0.129 & 0.017 & 0.072 \\
\hline 39.4 & 0.009 & 0.019 & 0.084 & 0.015 & 0.075 \\
\hline 45.0 & 0.008 & 0.018 & 0.057 & 0.016 & 0.077 \\
\hline 50.0 & 0.008 & 0.017 & 0.039 & 0.018 & 0.079 \\
\hline 54.3585 & 0.008 & 0.016 & 0.030 & 0.017 & 0.080 \\
\hline 60.0 & 0.008 & 0.016 & 0.029 & 0.017 & 0.084 \\
\hline 65.0 & 0.008 & 0.016 & 0.035 & 0.021 & 0.087 \\
\hline 70.0 & 0.008 & 0.016 & 0.043 & 0.027 & 0.091 \\
\hline 75.0 & 0.008 & 0.017 & 0.050 & 0.030 & 0.094 \\
\hline 80.0 & 0.008 & 0.017 & 0.056 & 0.031 & 0.097 \\
\hline 83.8058 & 0.008 & 0.018 & 0.060 & 0.031 & 0.100 \\
\hline
\end{tabular}


Table XII. NIST Type B uncertainties, $u_{13}$, and expanded uncertainties, $U_{\mathrm{T}}$, for comparison measurements of RIRTs between $0.65 \mathrm{~K}$ and $83.8 \mathrm{~K}$.

\begin{tabular}{|l|l|l|l|l||l|l|l|l|l|l|}
\hline \hline & \multicolumn{3}{|c|}{$\mathrm{He} \mathrm{VP} / \mathrm{lCVGT}$} & \multicolumn{2}{|c|}{ SPRT } & & \multicolumn{2}{|c|}{ ICVGT } & \multicolumn{2}{|c|}{ SPRT } \\
\hline$T_{90} / \mathrm{K}$ & $u_{\mathrm{B}} / \mathrm{mK}$ & $U_{\mathrm{T}} / \mathrm{mK}$ & $u_{\mathrm{B}} / \mathrm{mK}$ & $U_{\mathrm{T}} / \mathrm{mK}$ & $T_{90} / \mathrm{K}$ & $u_{\mathrm{B}} / \mathrm{mK}$ & $U_{\mathrm{T}} / \mathrm{mK}$ & $u_{\mathrm{B}} / \mathrm{mK}$ & $U_{\mathrm{T}} / \mathrm{mK}$ \\
\hline 0.65 & 0.076 & 0.154 & & & 18.66 & 0.129 & 0.263 & 0.109 & 0.224 \\
\hline 0.85 & 0.064 & 0.132 & & & 20.27 & 0.179 & 0.361 & 0.108 & 0.221 \\
\hline 1.18 & 0.061 & 0.124 & & & 21.5 & 0.137 & 0.278 & 0.103 & 0.211 \\
\hline 1.65 & 0.073 & 0.147 & & & 22.6 & 0.144 & 0.293 & 0.107 & 0.221 \\
\hline 2.2 & 0.040 & 0.081 & & & 23.6 & 0.147 & 0.306 & 0.119 & 0.244 \\
\hline 2.8 & 0.031 & 0.064 & & & 24.665 & 0.145 & 0.295 & 0.130 & 0.267 \\
\hline 3.414 & 0.039 & 0.082 & & & 26.0 & & & 0.144 & 0.293 \\
\hline 4.2 & 0.036 & 0.076 & & & 27.1 & & & 0.151 & 0.306 \\
\hline 5.1 & 0.070 & 0.142 & & & 31.2 & & & 0.151 & 0.303 \\
\hline 6.1 & 0.072 & 0.146 & & & 39.4 & & & 0.116 & 0.234 \\
\hline 7.2 & 0.076 & 0.155 & & & 45.0 & & & 0.099 & 0.199 \\
\hline 8.6 & 0.085 & 0.174 & & & 50.0 & & & 0.091 & 0.184 \\
\hline 10.2 & 0.090 & 0.183 & & & 54.358 & & & 0.089 & 0.179 \\
\hline 12.0 & 0.094 & 0.191 & & & 60.0 & & & 0.092 & 0.186 \\
\hline 13.8 & 0.103 & 0.208 & 0.103 & 0.209 & 65.0 & & & 0.098 & 0.197 \\
\hline 14.6 & 0.122 & 0.245 & 0.232 & 0.465 & 70.0 & & & 0.105 & 0.213 \\
\hline 15.4 & 0.117 & 0.236 & 0.223 & 0.448 & 75.0 & & & 0.112 & 0.226 \\
\hline 17.035 & 0.116 & 0.234 & 0.105 & 0.211 & 83.8 & & & 0.122 & 0.246 \\
\hline
\end{tabular}




\subsection{Conclusions}

The uncertainties given here represent a significant improvement in temperature metrology at NIST for the range below $83.8 \mathrm{~K}$ since the original NIST IR 5319 was prepared. Despite the fact that the uncertainties given in NIST IR 5319 did not include any estimates with respect to the actual ITS-90 definitions, the present uncertainty values provided here are in some cases less than those earlier evaluations.

This assessment provides the best evaluation of uncertainties for capsule SPRT and RIRT calibrations based on the data that have been generated as of August 1997 on the ITS-90 at NIST. As more realization data and comparison data become available, it is possible that some of the evaluations may change. This is particularly true of the dominant Type B contributions of $u_{3}$ and $u_{5}$. In fact, we fully expect that continual refinement of our techniques will reduce some of these most dominant uncertainty contributions. Every calibration report prepared at NIST will reflect the most recent evaluations for all the uncertainty parameters discussed here. While some uncertainty components may change for various reasons, the basic approach for treating the data outlined here will remain the same for the foreseeable future. 


\section{REFERENCES}

1. H. Preston-Thomas, "The International Temperature Scale of 1990 (ITS-90)", Metrologia 27, 3-10 (1990): ibid. p. 107.

2. "The International Practical Temperature Scale of 1968, Amended Edition of 1975", Metrologia, 12, 7-17 (1976).

3. "The 1976 Provisional 0.5 K to $30 \mathrm{~K}$ Temperature Scale", Metrologia, 15, 65-68 (1979).

4. B.W. Mangum and G.T. Furukawa, "Guidelines for Realizing the International Temperature Scale of 1990 (ITS-90)", NIST Technical Note 1265, 190 pp. (1990).

5. R.L. Rusby, "A Rhodium-Iron Resistance Thermometer for Use Below $20 \mathrm{~K}$ ", Temperature. Its Measurement and Control in Science and Industry, Edited by H.H. Plumb, Vol. 4, (Instrument Society of America, New York, 1972).

6. G.F. Strouse and W.L. Tew, "Assessment of Uncertainties of Calibration of Resistance Thermometers at the National Institute of Standards and Technology", NISTIR 5319, U.S. Department of Commerce, (January 1994).

7. B.N. Taylor and C.E. Kuyatt, "Guidelines for Evaluating and Expressing the Uncertainty of NIST Measurement Results", NIST Technical Note 1297, 1994 Edition., 20 pp. (September 1994).

8. ISO, "Guide to the Expression of Uncertainty in Measurement", International Organization for Standardization, Geneva, Switzerland (1993), (corrected and reprinted, 1995).

9. W.L. Tew and C. W. Meyer, "Recent Advances in the Realization and Dissemination of the ITS-90 below 83.8 K at NIST", in: Advances in Cryogenic Engineering, 43, pp. 733740, Plenum Press, (1997).

10. E.R. Pfeiffer, "Realization of the ITS-90 Below $83.8 \mathrm{~K}$ at the National Institute of Standards and Technology", Temperature. Its Measurement and Control in Science and Industry. Edited by J.F. Schooley, Vol. 6, (American Institute of Physics, New York, 1992).

11. R.L. Rusby, "The Rhodium-Iron Resistance Thermometer, Ten Years On", Temperature. Its Measurement and Control in Science and Industry, Edited by J.F. Schooley, Vol. 5, (American Institute of Physics, New York, 1982).

12. J.F. Schooley and R. J. Soulen, Jr., "Superconductive thermometric fixed points", Temperature. Its Measurement and Control in Science and Industry, Edited by J.F. Schooley, Vol. 5, (American Institute of Physics, New York, 1982).

13. F.J. Wilkins, and M.J. Swan, "Precision ac/dc resistance standards", Proc. IEE, Vol. 117, p. 841, (April, 1970).

14. G. F. Strouse and B.W. Mangum, "NIST Measurement Assurance of SPRT Calibrations on the ITS-90: A Quantitative Approach" Proceedings of the Measurement Science Conference, Anaheim, CA, January 1993, session 1-D.

15. G. T. Furukawa, J. L. Riddle, W. R. Bigge, E. R. Pfeiffer, "Application of Some Metal SRMs as Thermometric Fixed Points, NBS Special Publication 260-77, U.S. Department of Commerce, (August 1982).

16. G.F. Strouse, "NIST Implementation and Realization of the ITS-90 Over the Range $83.8 \mathrm{~K}$ to $1235 \mathrm{~K}$, Reproducibility, Stability, and Uncertainties.", Temperature. Its Measurement and Control in Science and Industry, Edited by J.F. Schooley, Vol. 6. pp. 169-174, (American Institute of Physics, New York, 1992). 
17. C.W. Meyer and M.L. Reilly, "A Progress Report on the Primary Realization of the International Temperature Scale of 1990 from $0.65 \mathrm{~K}$ to $83.8 \mathrm{~K}$ at the National Institute of Standards and Technology", Consultative Committee on Thermometry, (Bureau International des Poids et Mesures, Paris, 1993).

18. C.W. Meyer and M.L. Reilly, " Realization of the ITS-90 in the range $0.65 \mathrm{~K}$ to $5.0 \mathrm{~K}$ using vapor pressure thermometry", Metrologia, 33, p. 383, (1996).

19. C.W. Meyer and M.L. Reilly, "Realization of the ITS-90 in the range $3.0 \mathrm{~K}$ to $24.5561 \mathrm{~K}$ Using an Interpolating Constant Volume Gas Thermometer", TEMPMEKO 97 Conference Proceedings, Torino, September 1996, to be published, (1997).

20. W.L. Tew, "Sealed Cells for the Realization of Triple Points at NIST", TEMPMEKO 97 Conference Proceedings, Torino, September 1996, to be published, (1997).

21. W.L. Tew and B.W. Mangum, "New Capabilities and Procedures for the Calibration of Cryogenic Resistance Thermometers at NIST", Advances in Cryogenic Engineering, P. Kittel, ed., 39B, p.1019, (Plenum Press, 1994).

22. A.M. Thompson. "Precise Calibration of Ratio Transformers", IEEE Trans. Instrum. Meas., IM-32, pp 47-50, (1983).

23. Automatic Systems Laboratories, "Model F18 Operators Handbook: ASL-F18-14-015, Issue 1a", section 3.5, (Automatic Systems Laboratories, Wilmington, MA, 1992)

24. W.L. Tew and G.F. Strouse, "Maintenance and Validation of a Resistance Ratio Chain for Platinum Resistance Thermometry", submitted to: IEEE Trans. Instrum. Meas. (Proceedings of the Conference on Precision Electromagnetic Measurements, Washington, DC, July 1998).

25. D.R. White, K. Jones, J.M. Williams, and I.E. Ramsey. "A Simple Resistance Network for Calibrating Resistance Bridges ", IEEE Trans. Instrum. Meas., IM-46, pp 329-332, (1997).

26. R.F. Dziuba, P.A. Boynton, R.E. Elmquist, D.G. Jarrett, T.P. Moore, and J.D. Neal. NIST Measurement Service for DC Standard Resistors", NIST Technical Note 1298, U.S. Department of Commerce, (November 1992).

27. C.W. Meyer and M.L. Reilly, "Realization of the ITS-90 Triple Points Between $13.80 \mathrm{~K}$ to $83.81 \mathrm{~K}$ at NIST", Conference Proceedings of the IMEKO International Seminar on Low Temperature Thermometry and Dynamic Temperature Measurement, Wroclaw. Poland, to be published, (1998). 


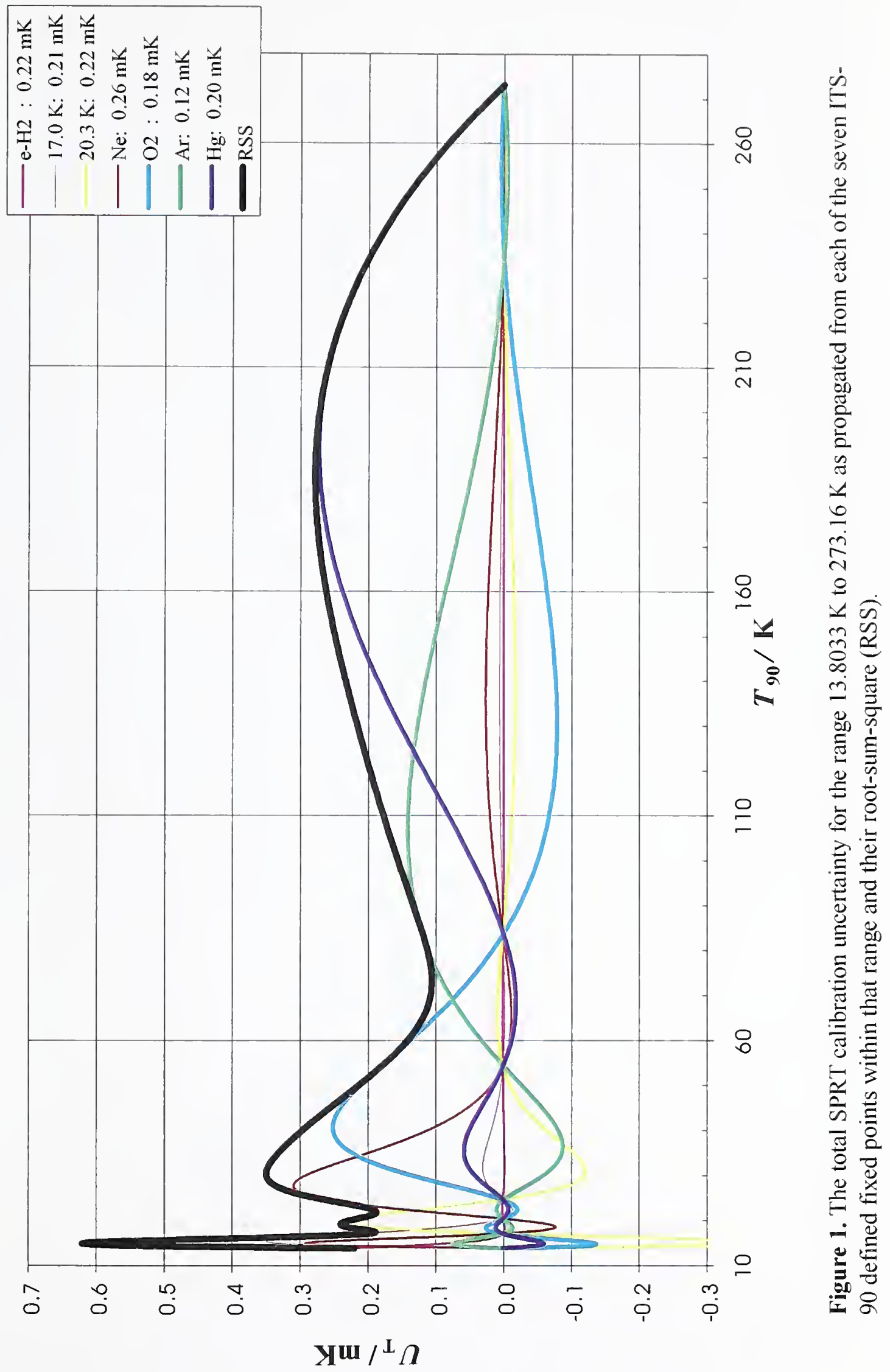





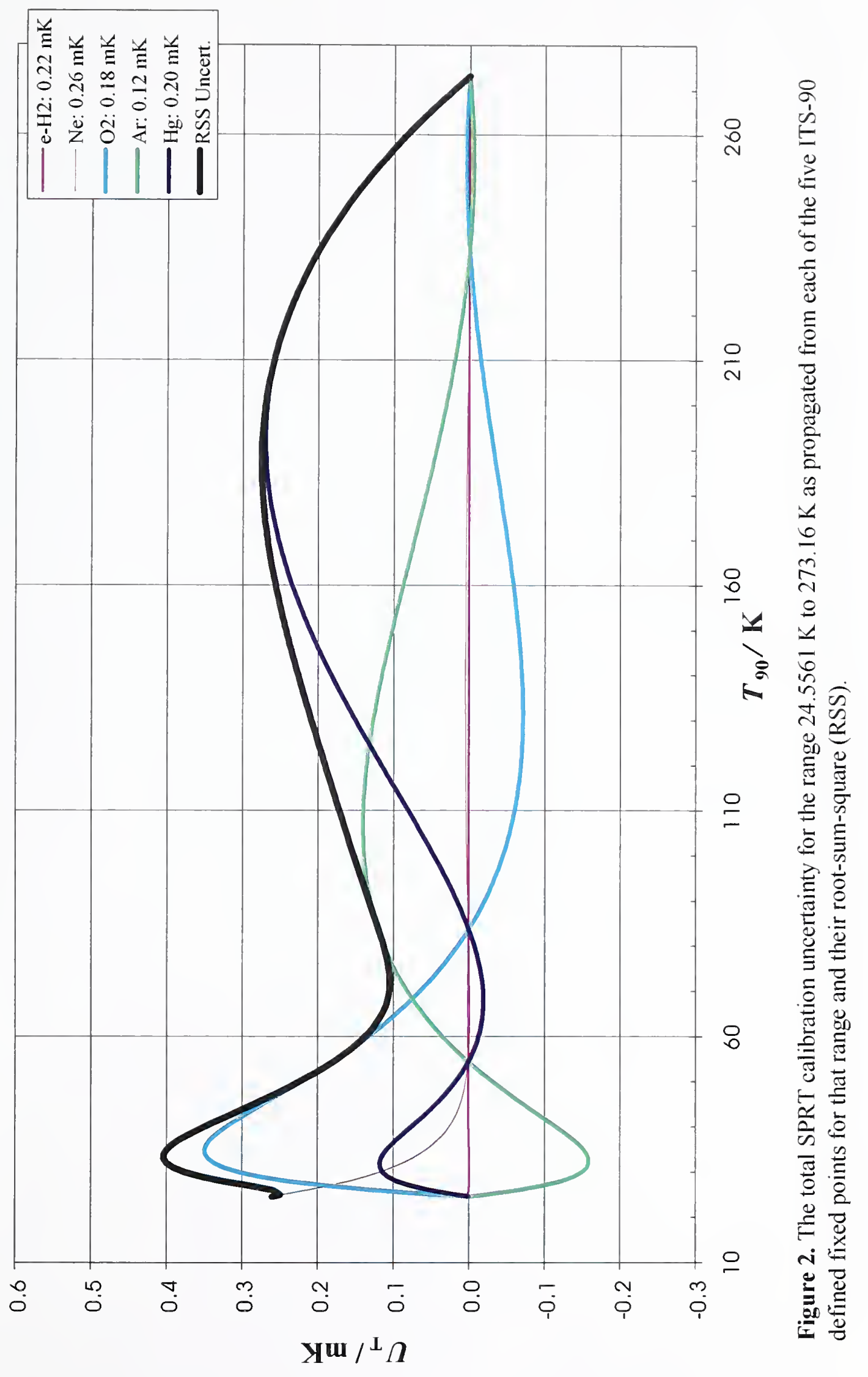





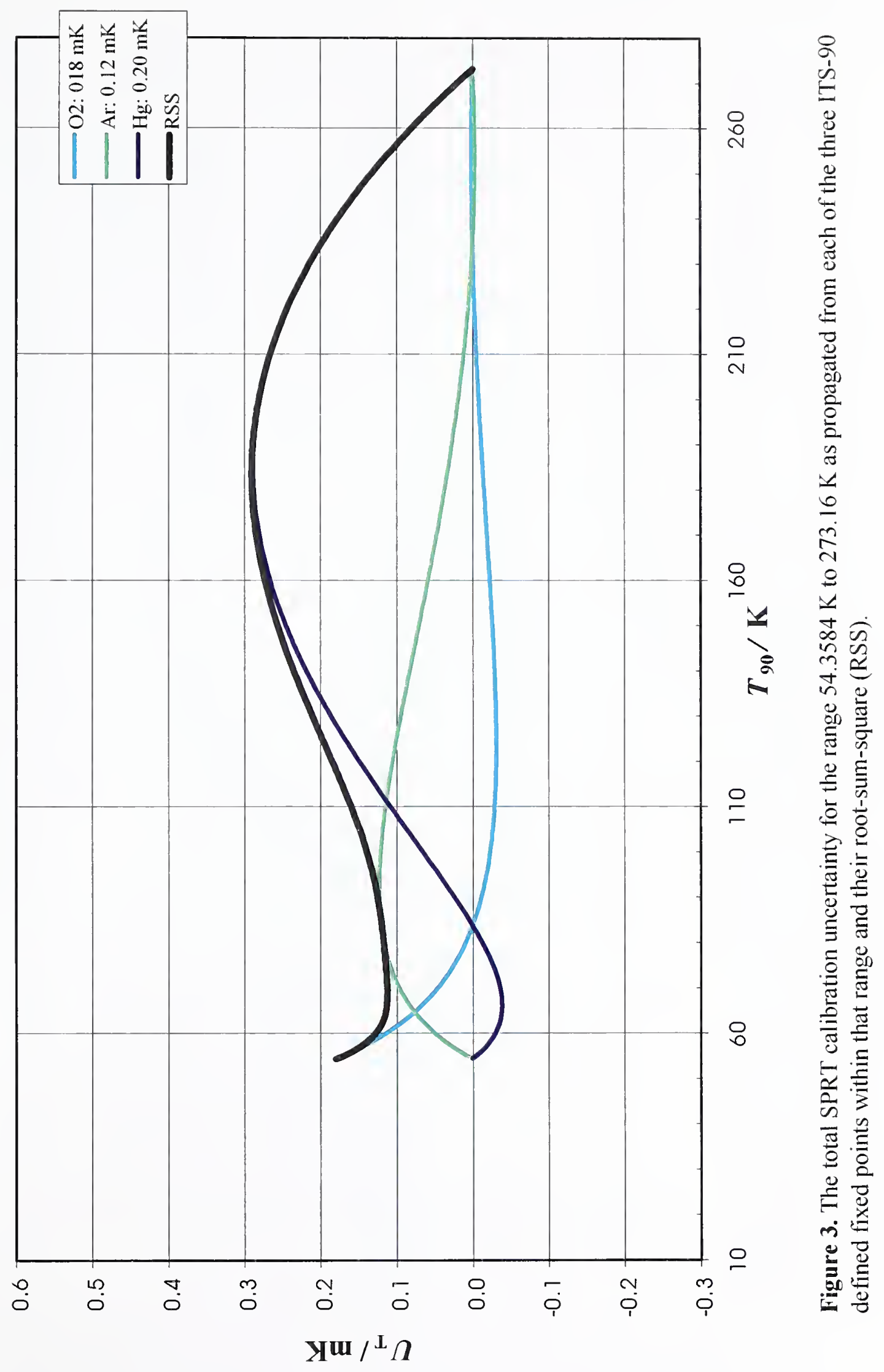





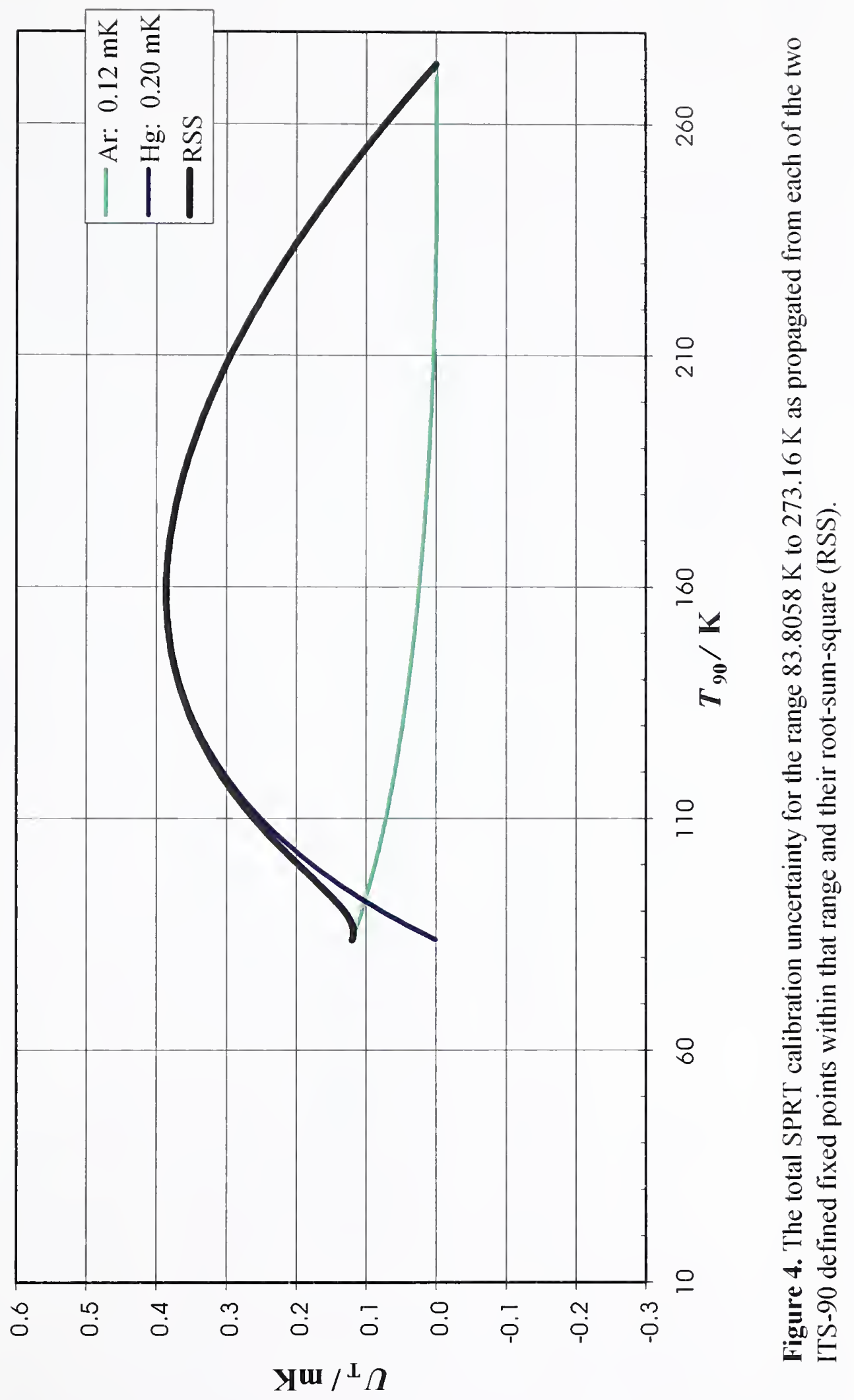





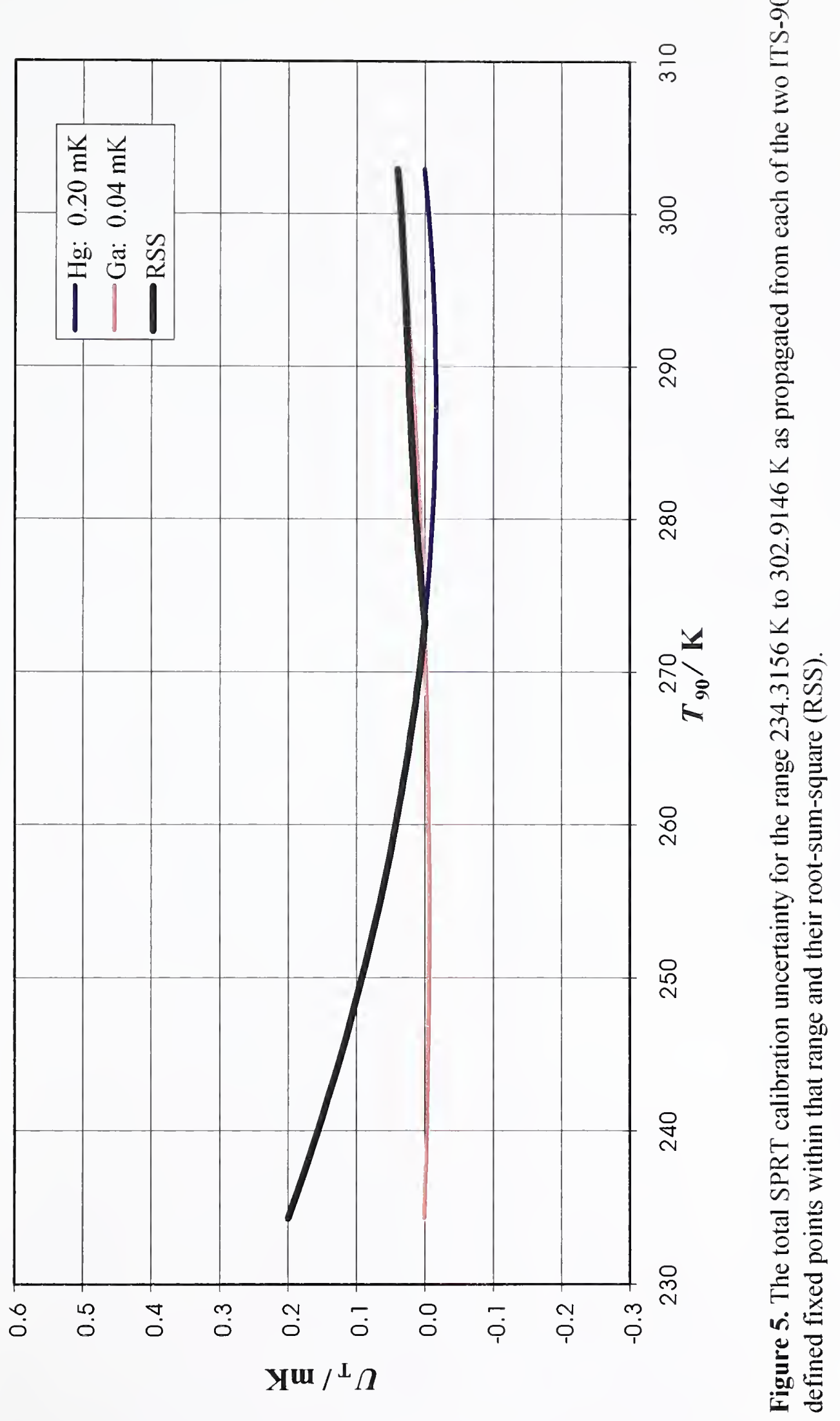





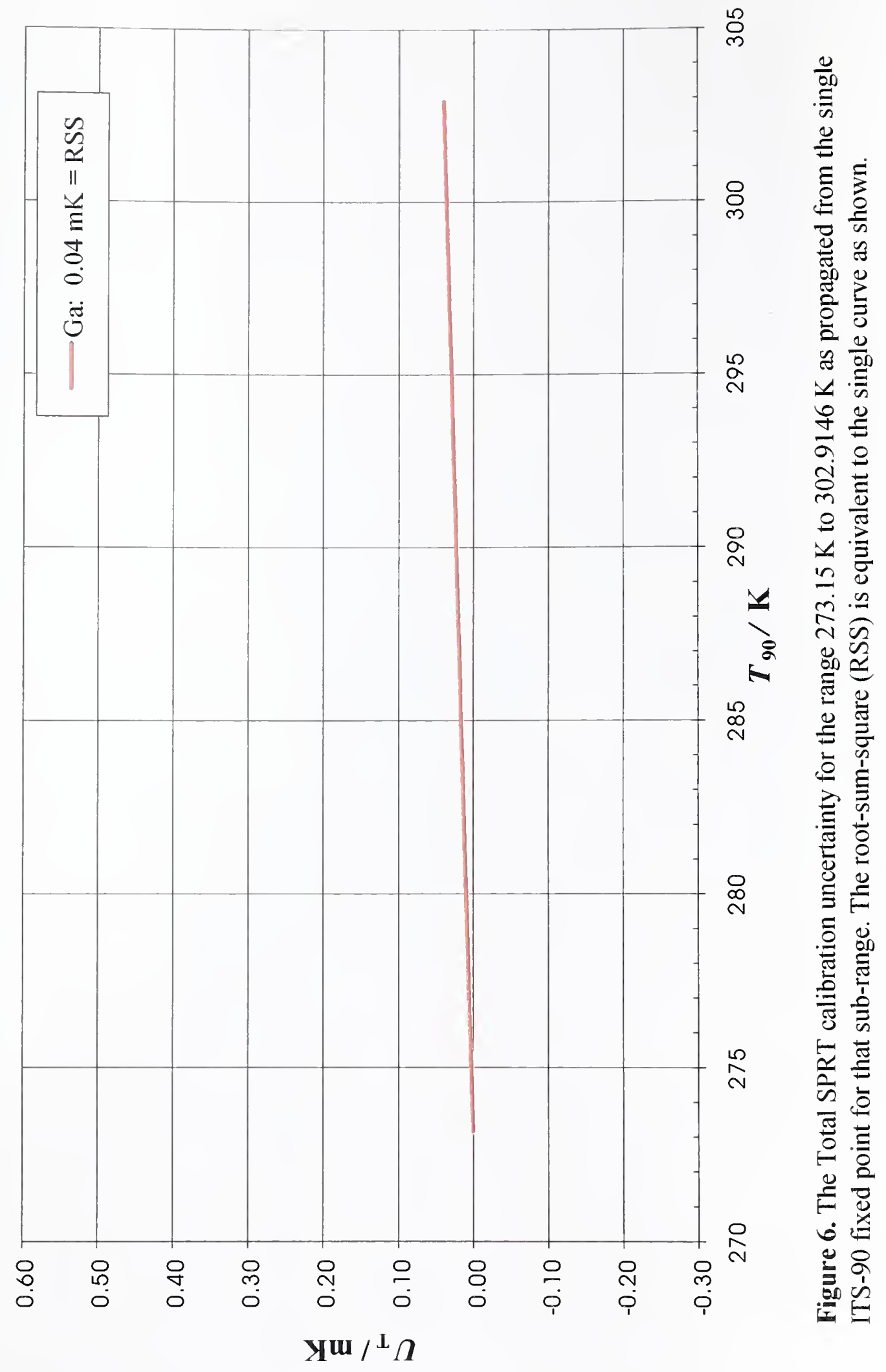





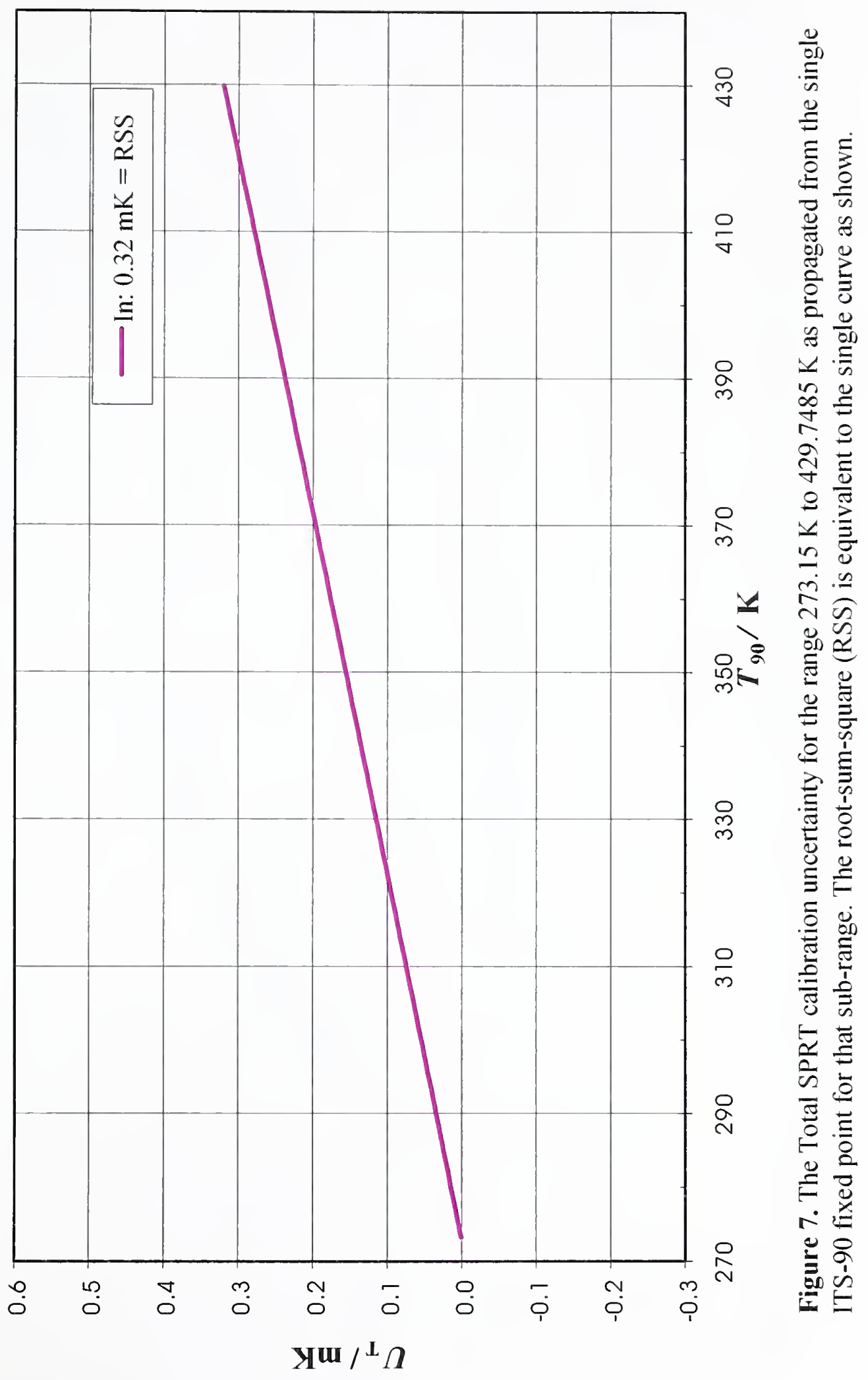





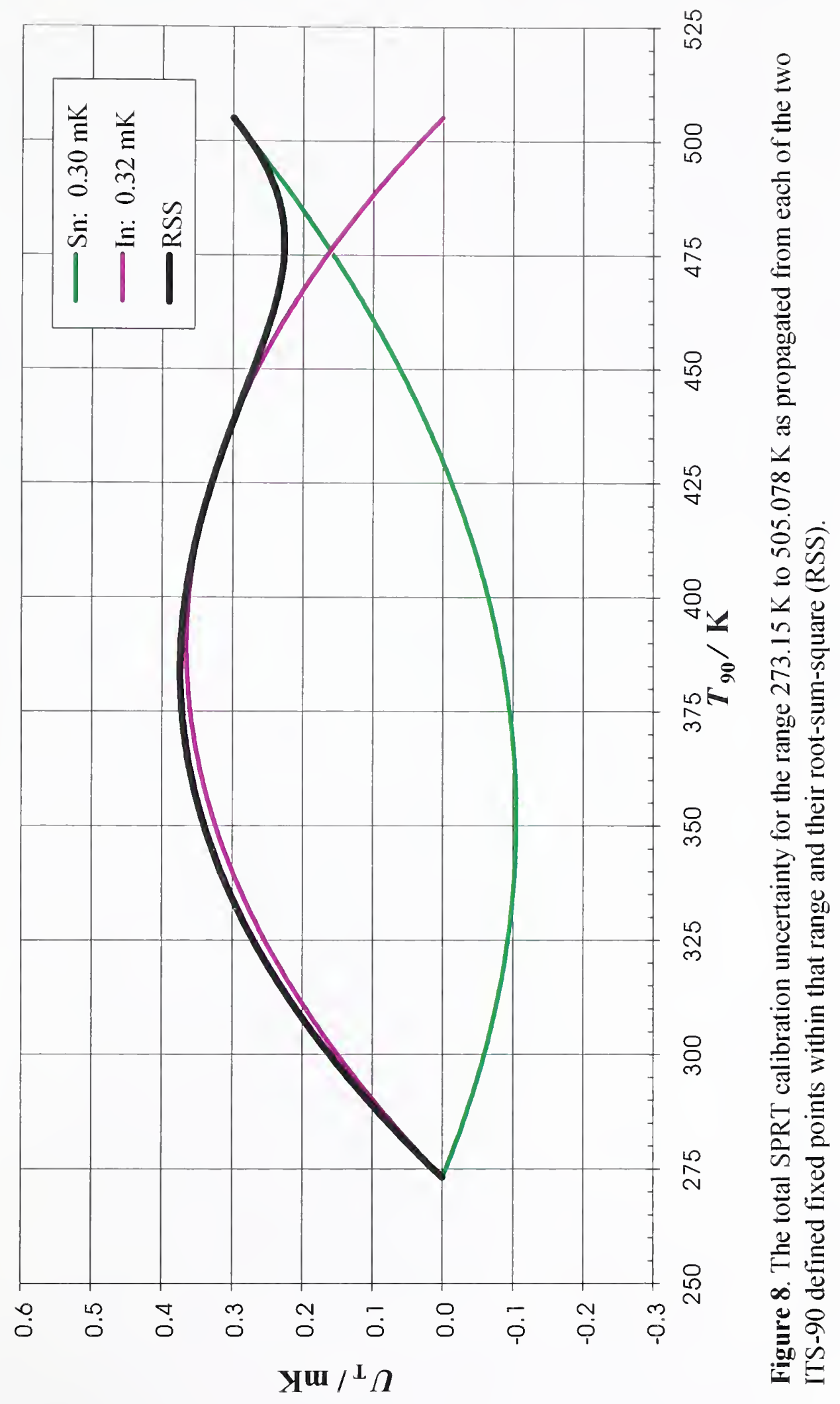




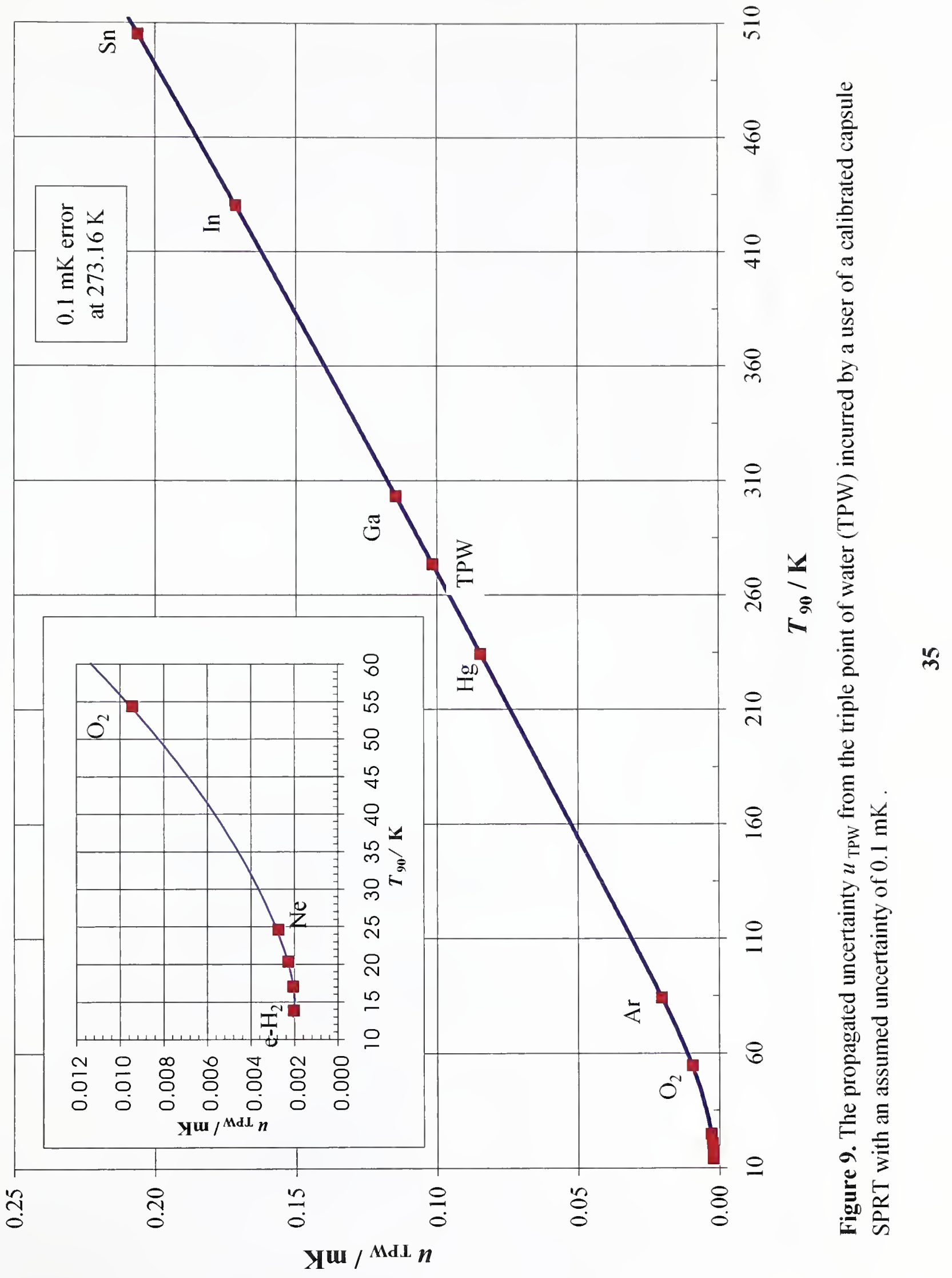





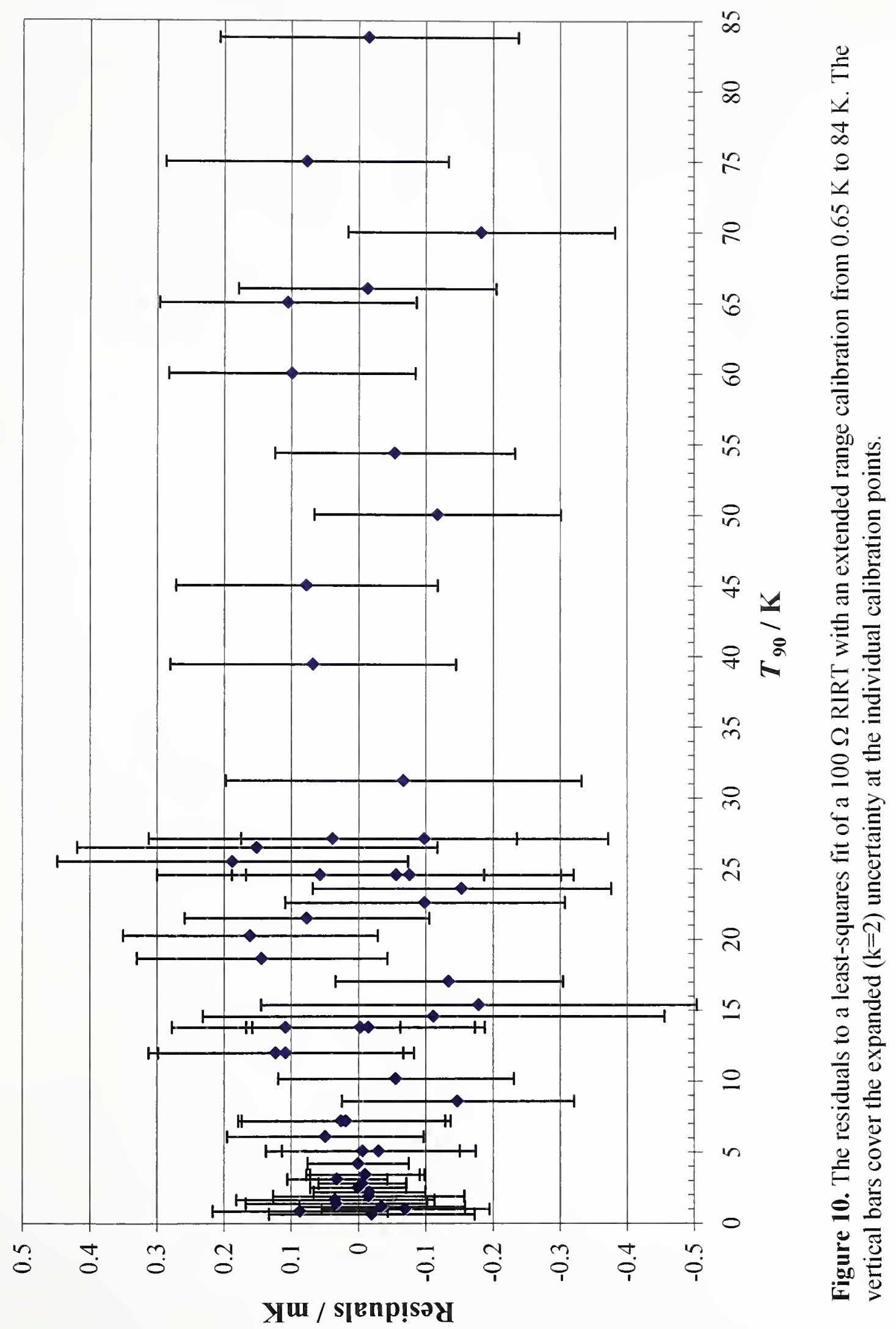






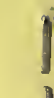

